\title{
Variational formulation of generalized interfaces for finite deformation elasticity
}

Mathematics and Mechanics of Solids 2018, Vol. 23(9) I303-1322 (C) The Author(s) 2017 Article reuse guidelines: sagepub.com/journals-permissions DOI: $10.1177 / 1081286517719938$ journals.sagepub.com/home/mms (S)AGE

\author{
Ali Javili \\ Department of Mechanical Engineering, Bilkent University, Ankara, Turkey
}

Received I3 April 2017; accepted 20 June 2017

\begin{abstract}
The objective of this contribution is to formulate generalized interfaces in a variationally consistent manner within a finite deformation continuum mechanics setting. The general interface model is a zero-thickness model that represents the finite thickness "interphase" between different constituents in a heterogeneous material. The interphase may be the transition zone between inclusion and matrix in composites or the grain boundaries in polycrystalline solids. The term "general" indicates that the interface model here accounts for both jumps of the deformation as well as the traction across the interface. Both the cohesive zone model and elastic interface model can be understood as two limits of the current interface model. Furthermore, some aspects of material modeling of generalized interfaces are elaborated and a consistent model is proposed. Finally, the proposed theory is elucidated via a series of numerical examples.
\end{abstract}

\section{Keywords}

General imperfect interface, variational elasticity, finite deformation, size effect

\section{Introduction}

Almost all materials at a certain scale of observation possess heterogeneous micro-structures. Homogenization pioneered by Hill $[1,2]$ and Ogden [3] has proven to be a powerful tool to link the overall material response at the macro-scale to the behavior of its constituents at the micro-scale. Computational homogenization is a mature field and has been thoroughly reviewed in [4-8]. In a heterogeneous micro-structure, the interphases between various constituents play a crucial role in the overall material response and cannot be neglected. The finite thickness interphase can be sufficiently approximated by a zero-thickness interface model. The main objective of this contribution is to formulate generalized interfaces in a variationally consistent manner. Note that the idea behind this contribution relies heavily on the seminal work of Hashin [9] where he distinguishes between perfect and imperfect interface models. Furthermore, McBride et al. [10] show that classical interface models cannot capture the response of heterogeneous material layers; see Figure 1. However, a distinct feature here is to formulate the general interface model from a variational perspective within a finite deformation setting and also to elucidate the theory using computational simulations. Undoubtedly, emerging applications of nano-materials will require better understanding of interfaces since the influence of lower-dimensional media on the overall material response increases with decreasing size. See Figure 2 for an illustration of a size effect due to the presence of interfaces.

Dedicated to the memory of Professor Emeritus Yakov Benveniste (23 December 1943 - 12 March 2015)

Corresponding author:

Ali Javili, Department of Mechanical Engineering, Bilkent University, 06800 Ankara, Turkey.

Email: ajavili@bilkent.edu 


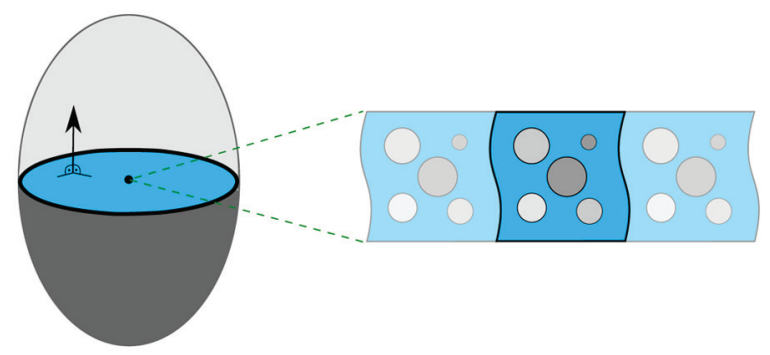

Figure I. Motivation for the need for a general interface model. Homogenization of heterogeneous material layers explains why even the simplest elastic response requires a generalized interface model to be properly captured [10]. The zero-thickness interface model (left) is representative of a finite thickness interphase (right).

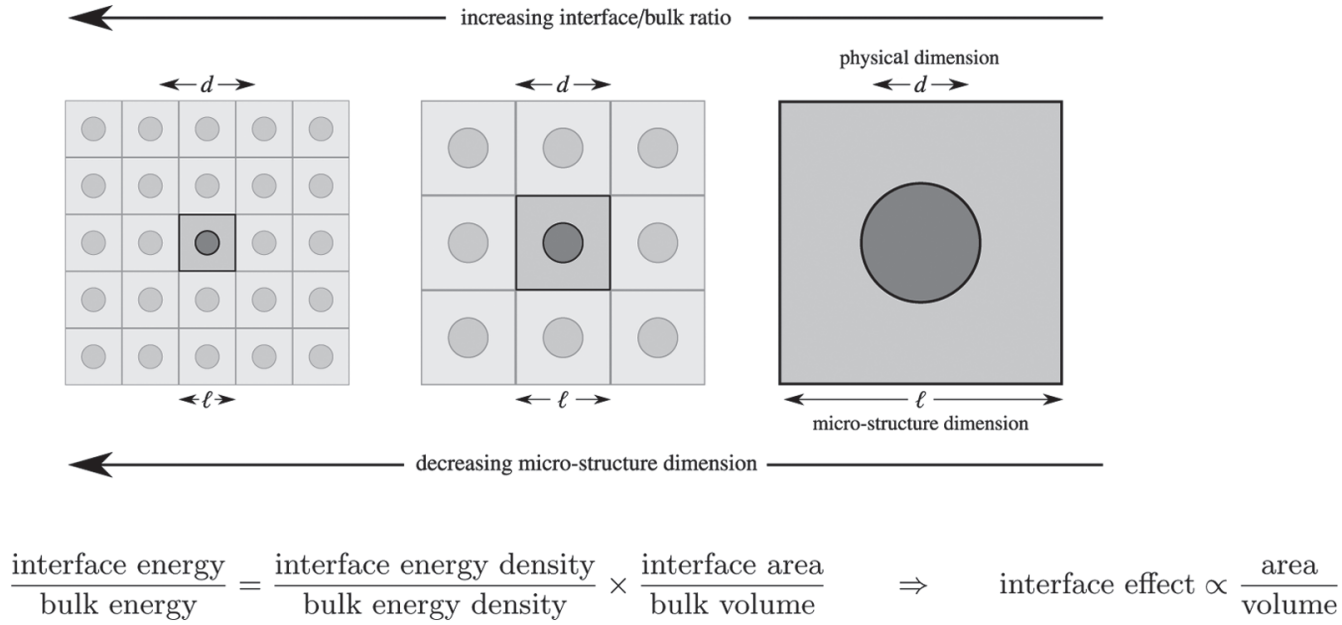

Figure 2. Illustration of the interplay between the micro-structure dimension $\ell$ and physical dimension $d$. Decreasing the microstructure dimension leads to an increase of the interface/bulk ratio and, hence, the interface effect on the overall material response. The ratio of the energy densities depends solely on the material, but the area-to-volume ratio is proportional to the inverse of the problem dimension. Note that an interface is a two-dimensional manifold in the three-dimensional embedding space. It is assumed that the (inclusion/matrix) volume ratio is identical in all three cases. In the absence of interfaces, all three cases result in the same effective material response.

\section{I.I. Literature review}

Interfaces can be categorized into four models according to the continuity of the displacement or traction field across the interface as shown in Figure 3. The perfect interface model, also referred to as free singular surfaces [11], does not allow for displacement jumps or traction jumps across the interface. The elastic interface model, also referred to as thermodynamic singular surfaces [11], is imperfect in the sense that the traction across the interface is not continuous, unlike the displacement field. The traction jump across the interface results from the divergence of the stress along the interface and follows the generalized Young-Laplace equation (see [12,13] among others). Interface elasticity theory [11,14-17] has its roots in the surface elasticity theory of Gurtin and Murdoch [18], which has been further investigated and extended in [19-34] among others. The cohesive interface model accounts for the displacement jump across the interface but the traction remains continuous and thus the cohesive interface model is only kinematically imperfect. The cohesive interface model is a mature field and dates back to the seminal works of Barenblatt [35, 36] and Dugdale [37] which have been extensively studied in [38-52] with various applications to decohesion, peeling and fracture from both theoretical and computational aspects. Obviously, the perfect interface model is the intersection of the two (semi-perfect) elastic and cohesive interface models; see Figure 3. The general imperfect interface model unifies all various types of interfaces and allows for jumps in both the displacement and the traction as shown in Figure 3. Unlike the cohesive and elastic interface models, the general interface model is not well-established to date with the exception of a few works dealing with analytical aspects of such interfaces [53-61] limited to 


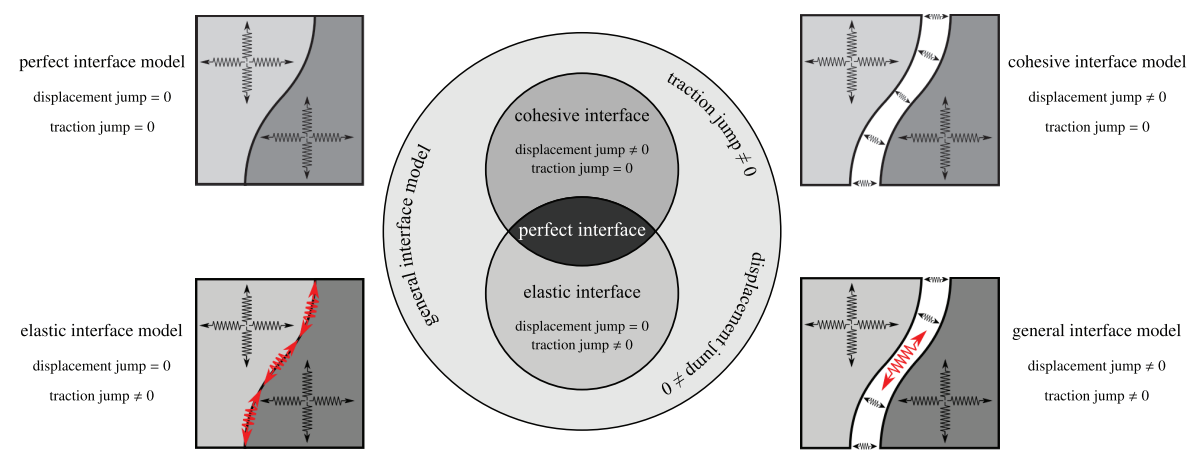

Figure 3. An overview of the interface models: coherent interface models (left) and non-coherent interface models (right). From the viewpoint of continuum mechanics, interfaces can be divided into four categories depending on the continuity of the displacement or traction across the interface. The elastic interface model does not allow for the displacement jump, but the traction may suffer a jump across the interface. The cohesive interface model on the contrary allows for the displacement jump but continuous traction. The intersection of the elastic and cohesive interface models is the perfect interface model for which both traction9 and displacements across the interface are continuous. This contribution formulates the general interface model which encompasses all other interface types.

small strains and derived from simplified asymptotic limits of thin interphases. From the perspective of deriving interface models as asymptotic limits of thin interphases, the cohesive interface model is derived as the limit case of soft interphases and is termed the spring interface model. In contrast, the elastic interface model is obtained as the limit case of stiff interphases and is termed the stress interface model.

The importance of the in-plane response of the interface can be realized via homogenization of heterogeneous material layers [10]. Related works on micro-to-macro transition of material layers, however, with no particular attention to the in-plane stretch of the interface, include [62-67]. Interface models can be related to the phase field approach through a thermodynamically consistent procedure [68]; see also [69-71] among others. For the kinetics of phase boundaries with edges and junctions, see $[72,73]$. Note that the proposed interface formulation here is variationally consistent which guarantees the thermodynamic consistency a priori. For a discussion on the thermodynamic consistency of cohesive models, see [74] and the references therein. Steinmann and Häsner [75] and Esmaeili et al. [76] formulated interfaces accounting for both displacement and traction jumps with extension to finite deformations and degradation. The main objective of this contribution is to formulate general imperfect interfaces at finite deformations in a variationally consistent manner.

\subsection{Organization of the manuscript}

This manuscript is organized as follows. Notation and definitions are briefly introduced and key features are highlighted. Section 2 elaborates on the variational formulation of general imperfect interfaces. The kinematics of the general interface model at finite deformation is formulated in Section 2.1 and the governing equations are derived in Section 2.2. Section 2.3 addresses the constitutive modeling and provides a specific interface free energy. Section 3 illustrates the influence of general interfaces on the material response via a series of numerical examples. Section 4 concludes this work and discusses its applications.

\section{I.3. Notation and definitions}

Quantities defined on the interface are distinguished from those in the bulk by a bar placed above the quantity. That is, $\{\bar{\bullet}\}$ refers to an interface variable with its bulk counterpart being $\{\bullet\}$. Throughout the manuscript, surface, interface and curve quantities are denoted as $\{\hat{\bullet}\},\{\bar{\bullet}\}$ and $\{\tilde{\bullet}\}$, respectively, and are therefore distinguished from the bulk quantity $\{\bullet\}$ by an accent on top of the quantity. Instead of the term "general imperfect interface model" its shorter variant "general interface model" is used occasionally. The term "traction jump" corresponds to the jump of the tractions across the interface at the reference configuration. The traction jump is the stress jump contracted with the interface normal and thereby both the stress and the normal are their respective values at the reference configuration. Direct notation is adopted throughout. The notation $\{\bullet\}:=\{\circ\}$, instead of an equality sign, is used occasionally to emphasize that the left-hand side $\{\bullet\}$ is defined by the 

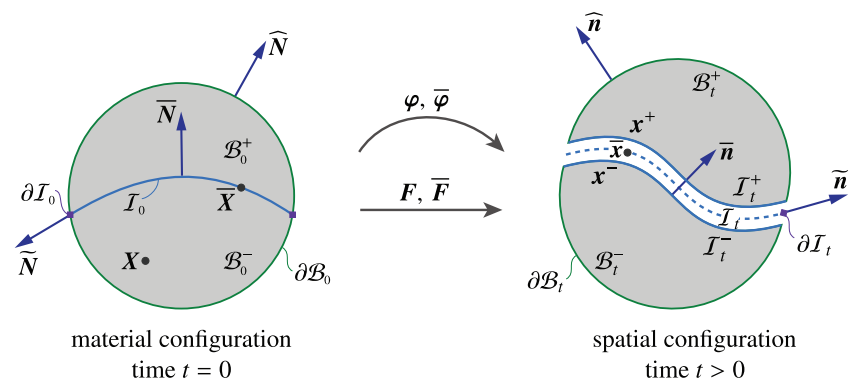

Figure 4. Motion of a continuum body including an interface under finite deformations. The illustration is made in two dimensions for clarity. The two sides of the body in the material configuration intersect at the interface $\mathcal{I}_{0}$. The interface is only a zero-thickness layer initially with $\llbracket \mathbf{X} \rrbracket=\mathbf{0}$, dividing the bulk into two sides $\mathcal{B}_{0}^{-}$and $\mathcal{B}_{0}^{+}$. Through the motion $\varphi$ the interface opens resulting in the displacement jump $\llbracket \mathbf{x} \rrbracket \neq \mathbf{0}$ across the interface.

expression on the right-hand side $\{\circ\}$. The average and jump of a quantity $\{\bullet\}$ over the interface are defined by $\left\{\{\lfloor\bullet\}\}:=\frac{1}{2}\left[\{\bullet\}^{+}+\{\bullet\}^{-}\right]\right.$and $\left.\mathbb{L}\{\bullet\}\right]=\{\bullet\}^{+}-\{\bullet\}^{-}$, respectively. The average and jump operators show the property $\llbracket\{\bullet\} \cdot\{0\} \rrbracket:=\llbracket\{\bullet\} \rrbracket \cdot\{\{\{0\}\}+\{\{\{\bullet\}\} \cdot \mathbb{L}\{0\} \rrbracket$.

\subsection{Key aspects and contributions}

The general interface model in this contribution reduces to the elastic interface model and the cohesive interface model at its two limits. While both elastic interfaces and cohesive interfaces are commonly accepted today, the general interface model at finite deformation remains elusive and poorly understood. To establish a unified variationally consistent framework to formulate the general interface model at finite deformation elasticity is the main contribution of this manuscript. A specific free energy is proposed that furnishes the constitutive response of the general interface model. Obviously, the proposed free energy recovers both the interface elasticity and cohesive zone models. The nature of the interface material parameters are elucidated via numerical examples. Note that the current framework can be readily simplified to capture the surface elasticity theory. That is, this manuscript renders a variationally consistent surface elasticity at finite deformation as well. It is accepted that the size effect due to surface elasticity in nano-porous materials is physically interpretable and agrees well with available data and experiments (see [77] among others).

\section{Generalized interfaces at finite deformations}

The purpose of this section is to formulate the theory of general imperfect interfaces in the context of mechanical problems at finite deformations. A key feature of the current formulation is that the governing equations are obtained in a variationally consistent framework. Detailed expositions on non-linear continuum mechanics can be found in [78-80] among others. Necessary concepts and terminologies corresponding to the differential geometry of interfaces are briefly reviewed in Appendix 1.

\section{I. Problem definition}

Let $\mathscr{B}$ denote a continuum body that takes the material configuration $\mathcal{B}_{0}$ at time $t=0$ and the spatial configuration $\mathcal{B}_{t}$ at any time $t>0$, as depicted in Figure 4. For the sake of simplicity, the analysis here is limited to quasi-static conditions and thus, the time parameter $t$ is merely a history parameter to order the sequence of events. The interface $\mathcal{I}_{0}$ splits the material configuration $\mathcal{B}_{0}$ into two disjoint subdomains $\mathcal{B}_{0}^{-}$and $\mathcal{B}_{0}^{+}$. The interface $\mathcal{I}_{0}$ is precisely a two-sided surface whose intersection with the boundaries $\partial \mathcal{B}_{0}^{-}$and $\partial \mathcal{B}_{0}^{+}$gives $\mathcal{I}_{0}^{-}$and $\mathcal{I}_{0}^{+}$, respectively. Figure 5 illustrates the motion and the evolution of its interface due to the motion. The three surfaces $\mathcal{I}_{0}, \mathcal{I}_{0}^{-}$and $\mathcal{I}_{0}^{+}$coincide in the material configuration, but are distanced from each other due to the motion $\varphi$ or, more precisely, the jump of the motion at the interface $\llbracket \varphi \rrbracket$. In the spatial configuration, the two sides of the interface $\mathcal{I}_{t}^{-}$and $\mathcal{I}_{t}^{+}$are well-defined and exactly follow the motion of $\mathcal{B}_{t}^{-}$and $\mathcal{B}_{t}^{+}$, respectively. However, the interface $\mathcal{I}_{t}$ can be defined arbitrarily. An intuitive, yet arguably meaningful, choice for the motion of the interface $\bar{\varphi}$ is to define the (fictitious) interface $\mathcal{I}_{t}$ as the mid-surface between the two sides $\mathcal{I}_{t}^{-}$and $\mathcal{I}_{t}^{+}$ and hence, $\bar{\varphi}:=\{\{\varphi\}\}$. ${ }^{1}$ 


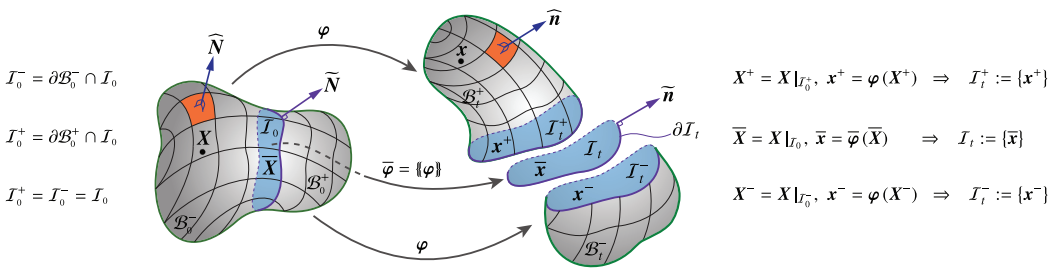

Figure 5. Material and spatial configurations of a continuum body embedding an interface. The interface $\mathcal{I}_{0}$ is identical to its two sides $\mathcal{I}_{0}^{-}$and $\mathcal{I}_{0}^{+}$in the material configuration. Due to the motion $\varphi$, the two sides of the interface are distanced from each other resulting in the gap $\llbracket \mathbf{x} \rrbracket \neq \mathbf{0}$ across the interface.

The placement of particles in the material configuration $\mathcal{B}_{0}$ is denoted $\boldsymbol{X}$ corresponding to particles in both $\mathcal{B}_{0}^{-}$and $\mathcal{B}_{0}^{+}$. Particles on the interface assume the placement $\bar{X}$ in the material configuration. Particles on $\mathcal{I}_{0}^{-}$and $\mathcal{I}_{0}^{+}$are denoted $\boldsymbol{X}^{-}$and $\boldsymbol{X}^{+}$, respectively. Obviously, the placements $\boldsymbol{X}^{-}$and $\boldsymbol{X}^{+}$in the material configuration coincide geometrically with $\bar{X}$. The motion $\varphi$ maps the placements of particles from the material configuration $\mathcal{B}_{0}$ to the spatial configuration $\mathcal{B}_{t}$ according to $\boldsymbol{x}=\boldsymbol{\varphi}(\boldsymbol{X})$ where $\boldsymbol{x}$ denotes the placement of particles in the spatial configuration $\mathcal{B}_{t}$. The non-linear deformation map $\varphi$ applies to the boundaries of the bulk, too. Therefore, the placements of particles on $\mathcal{I}_{0}^{-}$and $\mathcal{I}_{0}^{+}$are mapped onto their spatial counterparts $\boldsymbol{x}^{-}$and $\boldsymbol{x}^{+}$in $\mathcal{I}_{t}^{-}$and $\mathcal{I}_{t}^{+}$, respectively, according to $x^{-}=\varphi^{-}(\bar{X})$ and $x^{+}=\varphi^{+}(\bar{X})$. The motion of the interface $\bar{\varphi}$ maps the placement of particles on the interface from the material configuration $\mathcal{I}_{0}$ to the spatial configuration $\mathcal{I}_{t}$ according to

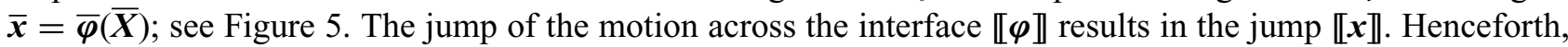
the motion jump across the interface is denoted as $\bar{f}:=\llbracket \varphi \rrbracket$ and the resultant displacement jump is denoted $\overline{\boldsymbol{y}}:=\llbracket \boldsymbol{x} \rrbracket$ to indicate its nature as an interface quantity independent of $\overline{\boldsymbol{x}}$. This notation furnishes the dual relations

$$
\overline{\boldsymbol{x}}=\bar{\varphi}(\bar{X}) \quad \text { and } \quad \bar{y}=\bar{f}(\bar{X}) \quad \text { with } \quad \bar{\varphi}:=\{\{\varphi\}\} \quad \text { and } \quad \bar{f}:=\llbracket \varphi \rrbracket \text {. }
$$

The surface $\mathcal{S}_{0}$ in the material configuration is composed of the boundary $\partial \mathcal{B}_{0}$ on both sides of the interface, but excluding the interface itself as $\mathcal{S}_{0}=\left[\partial \mathcal{B}_{0}^{-} \cup \partial \mathcal{B}_{0}^{+}\right] \backslash \mathcal{I}_{0}$. The boundary of the interface $\mathcal{I}_{0}$ in the material configuration is a curve defined as $\mathcal{C}_{0}:=\partial \mathcal{I}_{0}$. The bulk, surface, interface and curve in the spatial configuration are denoted $\mathcal{B}_{t}, \mathcal{S}_{t}, \mathcal{I}_{t}$ and $\mathcal{C}_{t}$, respectively. The outward unit normal to the surface $\mathcal{S}_{0}$ is denoted $\widehat{\boldsymbol{N}}$. The unit normal to the interface in the material configuration is denoted $\overline{\boldsymbol{N}}$ and is oriented such that it points from the minus side to the plus side of the interface. The outward unit normal to the boundary of the interface $\mathcal{C}_{0}$ but tangential to the interface $\mathcal{I}_{0}$ is denoted $\widetilde{N}$, shown in Figure 5. It is crucial in the derivations of the governing equations that neither the normal nor the bi-normal to the curve $\mathcal{C}_{0}$ in the sense of Freńet-Serret formula is the same as $\widetilde{N}$ and special care should be taken when computing $\widetilde{N}$. Furthermore, note $\widetilde{N}$ is not necessarily normal to the surface $\mathcal{S}_{0}$ as depicted in Figure 4. The unit normals in the spatial configuration are denoted $\widehat{\boldsymbol{n}}, \overline{\boldsymbol{n}}$ and $\widetilde{\boldsymbol{n}}$ on $\mathcal{S}_{t}, \mathcal{I}_{t}$ and $\mathcal{C}_{t}$, respectively.

The linear deformation maps associated with the non-linear deformation maps $\varphi$ and $\bar{\varphi}$ are denoted $\boldsymbol{F}$ and $\overline{\boldsymbol{F}}$, respectively, and relate the infinitesimal line elements $\mathrm{d} \boldsymbol{X}$ and $\mathrm{d} \overline{\boldsymbol{X}}$ in the material configuration to their spatial counterparts $\mathrm{d} \boldsymbol{x}$ and $\mathrm{d} \overline{\boldsymbol{x}}$, respectively. The deformation gradient $\boldsymbol{F}$ is related to the non-linear deformation map $\boldsymbol{\varphi}$ via the relation $\boldsymbol{F}=\operatorname{Grad} \boldsymbol{\varphi}$ based on the Taylor expansion of first order. Let $\overline{\boldsymbol{I}}=\boldsymbol{I}-\overline{\boldsymbol{N}} \otimes \overline{\boldsymbol{N}}$ denote the interface identity tensor that is also a projection to the interface. The interface deformation gradient $\overline{\boldsymbol{F}}$ is the projection of the deformation gradient $\operatorname{Grad} \overline{\boldsymbol{\varphi}}$ onto the interface as $\overline{\boldsymbol{F}}=\operatorname{Grad} \overline{\boldsymbol{\varphi}} \cdot \overline{\boldsymbol{I}}$. Alternatively, and preferably, one can define the interface deformation gradient $\overline{\boldsymbol{F}}$ using the interface gradient operator as $\overline{\boldsymbol{F}}=\overline{\operatorname{Grad}} \overline{\boldsymbol{\varphi}}$ according to 1 . It is of particular importance that the interface deformation gradient $\overline{\boldsymbol{F}}$ is superficial by definition. That is, $\overline{\boldsymbol{F}}$ holds the property $\overline{\boldsymbol{F}} \cdot \overline{\boldsymbol{N}}=\mathbf{0}$ which is clear from the relation $\overline{\boldsymbol{I}} \cdot \overline{\boldsymbol{N}}=\mathbf{0}$. Let $\mathrm{d} V$ and $\mathrm{d} v$ denote the volume elements of the bulk in the material and spatial configurations, respectively. Analogously, let $\mathrm{d} A$ and $\mathrm{d} a$ denote the area elements of the interface in the material and spatial configurations, respectively. Equation (2) summarizes the important relations on kinematics of a continuum body with an interface as

$$
\begin{array}{lllll}
\boldsymbol{x}=\boldsymbol{\varphi}(\boldsymbol{X}), & \mathrm{d} \boldsymbol{x}=\boldsymbol{F} \cdot \mathrm{d} \boldsymbol{X}, & \mathrm{d} v=J \mathrm{~d} V, & J:=\operatorname{Det} \boldsymbol{F} & \text { in } \mathcal{B}_{0}, \\
\overline{\boldsymbol{x}}=\overline{\boldsymbol{\varphi}}(\overline{\boldsymbol{X}}), & \mathrm{d} \overline{\boldsymbol{x}}=\overline{\boldsymbol{F}} \cdot \mathrm{d} \overline{\boldsymbol{X}}, & \mathrm{d} a=\bar{J} \mathrm{~d} A, & \bar{J}:=\overline{\operatorname{Det}} \overline{\boldsymbol{F}} & \text { on } \mathcal{I}_{0} .
\end{array}
$$


Finally, Nanson's formula on the interface $\mathrm{d} \boldsymbol{a}=J \boldsymbol{F}^{-\mathrm{t}} \cdot \mathrm{d} \boldsymbol{A}$ relates the vectorial area element $\mathrm{d} \boldsymbol{A}:=\mathrm{d} A \overline{\boldsymbol{N}}$ in the material configuration to its spatial counterpart $\mathrm{d} \boldsymbol{a}:=\mathrm{d} a \overline{\boldsymbol{n}}$. Nanson's formula on the surface $\mathcal{S}_{0}$ is formally identical to the one on the interface $\mathcal{I}_{0}$, but the area elements are $\mathrm{d} \boldsymbol{A}:=\mathrm{d} A \widehat{\boldsymbol{N}}$ and $\mathrm{d} \boldsymbol{a}:=\mathrm{d} a \widehat{\boldsymbol{n}}$. The line element $\mathrm{d} \boldsymbol{L}$ tangential to the interface and normal to the boundary of the interface in the material configuration maps to its spatial counterpart via the interface normal map $\overline{\operatorname{Cof}} \overline{\boldsymbol{F}}=\bar{J} \overline{\boldsymbol{F}}^{\text {-t }}$ as $\mathrm{d} \mathbf{1}=\overline{\operatorname{Cof}} \overline{\boldsymbol{F}} \cdot \mathrm{d} \boldsymbol{L}$ in which $\mathrm{d} \boldsymbol{L}:=\mathrm{d} L \tilde{\boldsymbol{N}}$ and $\mathrm{d} \mathbf{1}:=\mathrm{d} l \tilde{\boldsymbol{n}}$.

\subsection{Governing equations}

The objective of this section is to derive the governing equations of a continuum body accounting for the general interface in a variationally consistent framework. The choice of a variational structure is particularly helpful since it reveals the admissible form of external forces and tractions without prior knowledge or assumptions. This advantage is less significant for first-order continua as compared to higher-gradient theories; see for instance [81-90]. For first-order continua, the somewhat intuitive Cauchy cut process gives the same result as the variational framework. Nonetheless, the nature of tractions and the form of Cauchy postulate changes dramatically as soon as we are dealing with higher-gradient theories. The variational framework here paves the way to extending the proposed framework to higher-gradient continua. Also, the variational approach furnishes the weak form with no extra effort or derivations. The weak form is central to the numerical simulations using the finite element method. Another advantage of the variational framework is that the derivations are substantially less burdensome compared to the approaches based on Cauchy postulates. It is possible to derive the governing equations starting from working [91] and then imposing the invariance with respect to superposed rigid body motions. The derivations here are shorter and somewhat more elegant especially when dealing with general interfaces. Finally, deriving the balance equations via a variational framework provides a suitable platform for constructing a variationally consistent homogenization framework.

In order to obtain the governing equations, the total energy functional is minimized. The total energy functional $\Psi^{\text {tot }}$ consists of the internal and external contributions denoted $\Psi_{\text {int }}^{\text {tot }}$ and $\Psi_{\text {ext }}^{\text {tot }}$, respectively. To minimize $\Psi^{\text {tot }}$ its first variation is set to zero as

$$
\Psi^{\text {tot }}=\Psi_{\text {int }}^{\text {tot }}+\Psi_{\text {ext }}^{\text {tot }} \quad \text { and } \quad \delta \Psi^{\text {tot }} \doteq 0 \Rightarrow \delta \Psi_{\text {int }}^{\text {tot }}+\delta \Psi_{\text {ext }}^{\text {tot }} \doteq 0 .
$$

Sections 2.2.1 and 2.2.2 elaborate on the contributions from the internal and external energies, respectively. Section 2.2.3 combines the findings of Sections 2.2.1 and 2.2.2 and provides the strong (local) form of the balance equations.

2.2.I. Internal energy. The total internal energy $\Psi_{\text {int }}^{\text {tot }}$ consists of the internal energies in the bulk and on the interface

$$
\Psi_{\text {int }}^{\text {tot }}=\Psi_{\text {int }}+\bar{\Psi}_{\text {int }},
$$

in which $\Psi_{\text {int }}$ and $\bar{\Psi}_{\text {int }}$ denote the bulk and the interface internal energies, respectively. Let $\psi_{\text {int }}$ and $\bar{\psi}_{\text {int }}$ denote the internal energy densities in the bulk and on the interface, respectively. The internal energies in the bulk and on the interface are the integrals of their corresponding internal energy densities over their associated domains as

$$
\Psi_{\text {int }}=\int_{\mathcal{B}_{0}} \psi_{\text {int }} \mathrm{d} V \quad \text { and } \quad \bar{\Psi}_{\text {int }}=\int_{\mathcal{I}_{0}} \bar{\psi}_{\text {int }} \mathrm{d} A
$$

The first variation of the total internal energy consists of the contributions from the bulk and interface as

$$
\delta \Psi_{\text {int }}^{\text {tot }}=\delta \Psi_{\text {int }}+\delta \bar{\Psi}_{\text {int }} .
$$

The internal energy density of the bulk $\psi$ is exactly the well-established free energy and is a function of the deformation gradient $\boldsymbol{F}$. This contribution focuses on (hyper-)elasticity and thus no dissipation is associated with the deformation process. Similar to the bulk, the arguments of the interface energy are the interface deformation gradient $\overline{\boldsymbol{F}}$ and the motion jump across the interface $\overline{\boldsymbol{f}}$. The bulk and the interface energy densities read

$$
\psi_{\mathrm{int}}=\psi_{\mathrm{int}}(\boldsymbol{F}) \quad \text { and } \quad \bar{\psi}_{\mathrm{int}}=\bar{\psi}_{\mathrm{int}}(\overline{\boldsymbol{F}}, \overline{\boldsymbol{f}}) .
$$


Dependence of the interface energy on $\overline{\boldsymbol{F}}$ for vanishing $\overline{\boldsymbol{f}}$ captures the interface elasticity theory. On the other hand, the cohesive interface model is recovered for $\overline{\boldsymbol{F}}=\overline{\boldsymbol{I}}$.

Bulk. The first variation of the bulk internal energy $\delta \Psi_{\text {int }}$ reads

$$
\delta \Psi_{\text {int }}=\delta \int_{\mathcal{B}_{0}} \psi_{\text {int }} \mathrm{d} V=\int_{\mathcal{B}_{0}} \frac{\partial \psi_{\text {int }}}{\partial \boldsymbol{F}}: \delta \boldsymbol{F} \mathrm{d} V=\int_{\mathcal{B}_{0}} \boldsymbol{P}: \delta \boldsymbol{F} \mathrm{d} V \quad \text { with } \quad \boldsymbol{P}:=\frac{\partial \psi_{\text {int }}}{\partial \boldsymbol{F}},
$$

where $\boldsymbol{P}$ is the Piola stress tensor. ${ }^{2}$ Considering that $\delta \boldsymbol{F}=\operatorname{Grad} \delta \boldsymbol{\varphi}$ and using the identity

$$
\boldsymbol{P}: \operatorname{Grad} \delta \boldsymbol{\varphi}=\operatorname{Div}(\delta \boldsymbol{\varphi} \cdot \boldsymbol{P})-\delta \boldsymbol{\varphi} \cdot \operatorname{Div} \boldsymbol{P}
$$

the variation of the bulk internal energy reads

$$
\delta \Psi_{\text {int }}=\int_{\partial \mathcal{B}_{0}} \delta \boldsymbol{\varphi} \cdot[\boldsymbol{P} \cdot \widehat{\boldsymbol{N}}] \mathrm{d} A-\int_{\mathcal{B}_{0}} \delta \boldsymbol{D} \cdot \operatorname{Div} \boldsymbol{P} \mathrm{d} V .
$$

Interface. The first variation of the interface internal energy $\delta \bar{\Psi}_{\text {int }}$ reads

$$
\delta \bar{\Psi}_{\text {int }}=\delta \int_{\mathcal{I}_{0}} \bar{\psi}_{\text {int }} \mathrm{d} A=\int_{\mathcal{I}_{0}} \frac{\partial \bar{\psi}_{\text {int }}}{\partial \overline{\boldsymbol{F}}}: \delta \overline{\boldsymbol{F}}+\frac{\partial \bar{\psi}_{\text {int }}}{\partial \overline{\boldsymbol{f}}}: \delta \overline{\boldsymbol{f}} \mathrm{d} A .
$$

Let $\overline{\boldsymbol{P}}$ denote the interface superficial stress tensor and $\overline{\boldsymbol{t}}$ denote the interface traction defined by

$$
\overline{\boldsymbol{P}}:=\frac{\partial \bar{\psi}_{\text {int }}}{\partial \overline{\boldsymbol{F}}} \quad \text { and } \quad \overline{\boldsymbol{t}}:=\frac{\partial \bar{\psi}_{\mathrm{int}}}{\partial \overline{\boldsymbol{f}}} .
$$

The variation of the interface internal energy can be written as

$$
\delta \bar{\Psi}_{\text {int }}=\int_{\mathcal{I}_{0}} \overline{\boldsymbol{P}}: \overline{\operatorname{Grad}} \delta \overline{\boldsymbol{\varphi}}+\overline{\boldsymbol{t}} \cdot \delta \overline{\boldsymbol{f}} \mathrm{d} A=\int_{\mathcal{I}_{0}} \overline{\operatorname{Div}}(\delta \overline{\boldsymbol{\varphi}} \cdot \overline{\boldsymbol{P}})-\delta \overline{\boldsymbol{\varphi}} \cdot \overline{\operatorname{Div}} \overline{\boldsymbol{P}}+\overline{\boldsymbol{t}} \cdot \delta \overline{\boldsymbol{f}} \mathrm{d} A .
$$

Next, the result of the interface divergence theorem

$$
\int_{\mathcal{I}_{0}} \overline{\operatorname{Div}}(\delta \overline{\boldsymbol{\varphi}} \cdot \overline{\boldsymbol{P}}) \mathrm{d} A=\int_{\mathcal{C}_{0}} \delta \overline{\boldsymbol{\varphi}} \cdot[\overline{\boldsymbol{P}} \cdot \tilde{\boldsymbol{N}}] \mathrm{d} L-\int_{\mathcal{I}_{0}} \bar{K} \delta \overline{\boldsymbol{\varphi}} \cdot \overline{\boldsymbol{P}} \cdot \overline{\boldsymbol{N}} \mathrm{d} A
$$

is inserted in equation (13) whereby the term containing the interface curvature $\bar{K}$ vanishes due to the superficiality property $\overline{\boldsymbol{P}} \cdot \overline{\boldsymbol{N}}=\mathbf{0}$ associated with the interface stress. Therefore, the variation of the interface internal energy reads

$$
\delta \bar{\Psi}_{\text {int }}=\int_{\mathcal{C}_{0}} \delta \overline{\boldsymbol{\varphi}} \cdot[\overline{\boldsymbol{P}} \cdot \tilde{\boldsymbol{N}}] \mathrm{d} L-\int_{\mathcal{I}_{0}} \delta \overline{\boldsymbol{\varphi}} \cdot \overline{\operatorname{Div}} \overline{\boldsymbol{P}}-\overline{\boldsymbol{t}} \cdot \delta \overline{\boldsymbol{f}} \mathrm{d} A .
$$

Bulk and interface combined. Next, the variation of the bulk internal energy (10) and the variation of the interface internal energy (15) are replaced in the total internal energy variation (6) as

$$
\delta \Psi_{\text {int }}^{\text {tot }}=\int_{\partial \mathcal{B}_{0}} \delta \boldsymbol{\varphi} \cdot[\boldsymbol{P} \cdot \widehat{\boldsymbol{N}}] \mathrm{d} A-\int_{\mathcal{B}_{0}} \delta \boldsymbol{\varphi} \cdot \operatorname{Div} \boldsymbol{P} \mathrm{d} V+\int_{\mathcal{C}_{0}} \delta \overline{\boldsymbol{\varphi}} \cdot[\overline{\boldsymbol{P}} \cdot \tilde{\boldsymbol{N}}] \mathrm{d} L-\int_{\mathcal{I}_{0}} \delta \overline{\boldsymbol{\varphi}} \cdot \overline{\operatorname{Div}} \overline{\boldsymbol{P}}-\overline{\boldsymbol{t}} \cdot \delta \overline{\boldsymbol{f}} \mathrm{d} A
$$

The first integral over $\partial \mathcal{B}_{0}$ shall be further simplified. Since $\partial \mathcal{B}_{0}=\partial \mathcal{B}_{0}^{-} \cup \partial \mathcal{B}_{0}^{+}=\mathcal{S}_{0} \cup \mathcal{I}_{0}^{-} \cup \mathcal{I}_{0}^{+}$, the integral over $\partial \mathcal{B}_{0}$ decomposes into three integrals as

$$
\int_{\partial \mathcal{B}_{0}} \delta \varphi \cdot[\boldsymbol{P} \cdot \widehat{\boldsymbol{N}}] \mathrm{d} A=\int_{\mathcal{S}_{0}} \delta \varphi \cdot[\boldsymbol{P} \cdot \widehat{N}] \mathrm{d} A+\int_{\mathcal{I}_{0}^{-}} \delta \varphi \cdot[\boldsymbol{P} \cdot \widehat{N}] \mathrm{d} A+\int_{\mathcal{I}_{0}^{+}} \delta \varphi \cdot[\boldsymbol{P} \cdot \widehat{\boldsymbol{N}}] \mathrm{d} A .
$$

The outward surface unit normal $\widehat{N}$ on the minus side of the interface $\mathcal{I}_{0}^{-}$coincides with the interface unit normal $\overline{\boldsymbol{N}}$ as $\left.\widehat{\boldsymbol{N}}\right|_{\mathcal{I}_{0}^{-}}=\overline{\boldsymbol{N}}$. In contrast, on the plus side of the interface $\mathcal{I}_{0}^{+}$the surface unit normal $\widehat{\boldsymbol{N}}$ is opposite 
the interface unit normal $\overline{\boldsymbol{N}}$ and thus $\left.\widehat{\boldsymbol{N}}\right|_{\mathcal{I}_{0}^{+}}=-\overline{\boldsymbol{N}}$. Therefore, the integral over $\partial \mathcal{B}_{0}$ results in one term on the external surface $\mathcal{S}_{0}$ and a jump term on the interface $\mathcal{I}_{0}$ as

$$
\int_{\partial \mathcal{B}_{0}} \delta \boldsymbol{\varphi} \cdot[\boldsymbol{P} \cdot \widehat{\boldsymbol{N}}] \mathrm{d} A=\int_{\mathcal{S}_{0}} \delta \boldsymbol{\varphi} \cdot[\boldsymbol{P} \cdot \widehat{\boldsymbol{N}}] \mathrm{d} A-\int_{\mathcal{I}_{0}} \llbracket \delta \boldsymbol{P} \cdot \boldsymbol{P} \rrbracket \cdot \overline{\boldsymbol{N}} \mathrm{d} A .
$$

Inserting equation (18) into equation (16) yields

$$
\begin{aligned}
\delta \Psi_{\text {int }}^{\text {tot }}=- & \int_{\mathcal{B}_{0}} \delta \boldsymbol{\varphi} \cdot \operatorname{Div} \boldsymbol{P} \mathrm{d} V+\int_{\mathcal{S}_{0}} \delta \boldsymbol{\varphi} \cdot[\boldsymbol{P} \cdot \widehat{\boldsymbol{N}}] \mathrm{d} A \\
& -\int_{\mathcal{I}_{0}} \delta \overline{\boldsymbol{\varphi}} \cdot \overline{\operatorname{Div}} \overline{\boldsymbol{P}}-\overline{\boldsymbol{t}} \cdot \delta \overline{\boldsymbol{f}}+\llbracket \delta \boldsymbol{\varphi} \cdot \boldsymbol{P} \rrbracket \cdot \overline{\boldsymbol{N}} \mathrm{d} A+\int_{\mathcal{C}_{0}} \delta \overline{\boldsymbol{\varphi}} \cdot[\overline{\boldsymbol{P}} \cdot \tilde{\boldsymbol{N}}] \mathrm{d} L .
\end{aligned}
$$

To proceed, the identity

$$
\llbracket \delta \varphi \cdot P \rrbracket \cdot \bar{N}=\llbracket \delta \varphi \rrbracket \cdot\{\{P\}] \cdot \bar{N}+\{\{\delta \varphi\}\} \cdot \llbracket P \rrbracket \cdot \bar{N}=\delta \bar{f} \cdot\{\{P\}] \cdot \bar{N}+\delta \bar{\varphi} \cdot \llbracket P \rrbracket \cdot \bar{N}
$$

is employed in equation (19). After some mathematical steps, the variation of the total internal energy $\Psi_{\text {int }}^{\text {tot }}$ is recast into the integrals over the body $\mathcal{B}_{0}$, surface $\mathcal{S}_{0}$, interface $\mathcal{I}_{0}$ and curve $\mathcal{C}_{0}$ as

$$
\begin{aligned}
\delta \Psi_{\text {int }}^{\mathrm{tot}}=- & \int_{\mathcal{B}_{0}} \delta \boldsymbol{\varphi} \cdot \operatorname{Div} \boldsymbol{P} \mathrm{d} V+\int_{\mathcal{S}_{0}} \delta \boldsymbol{\varphi} \cdot[\boldsymbol{P} \cdot \widehat{\boldsymbol{N}}] \mathrm{d} A \\
& -\int_{\mathcal{I}_{0}} \delta \overline{\boldsymbol{\varphi}} \cdot[\overline{\operatorname{Div}} \overline{\boldsymbol{P}}+\llbracket \boldsymbol{P} \rrbracket \cdot \overline{\boldsymbol{N}}] \mathrm{d} A+\int_{\mathcal{I}_{0}} \delta \overline{\boldsymbol{f}} \cdot[\overline{\boldsymbol{t}}-\{\{\boldsymbol{P}\}] \cdot \overline{\boldsymbol{N}}] \mathrm{d} A+\int_{\mathcal{C}_{0}} \delta \overline{\boldsymbol{\varphi}} \cdot[\overline{\boldsymbol{P}} \cdot \tilde{\boldsymbol{N}}] \mathrm{d} L .
\end{aligned}
$$

Obviously, the internal energy of the interface varies due to two independent families of deformations. The first interface integral indicates that $[\overline{\operatorname{Div}} \overline{\boldsymbol{P}}+\llbracket \boldsymbol{P} \rrbracket \cdot \overline{\boldsymbol{N}}]$ is energetically conjugate to the interface motion $\overline{\boldsymbol{\varphi}}$. Recall, the interface (average) motion $\bar{\varphi}$ is independent of the interface motion jump $\bar{f}$. Furthermore, the contribution $[\overline{\boldsymbol{t}}-\{\{\boldsymbol{P}\}] \cdot \overline{\boldsymbol{N}}]$ is energetically conjugate to the interface motion jump $\overline{\boldsymbol{f}}$. In view of equation (21), it is of particular interest to note that the variation of the total internal energy $\Psi_{\text {int }}^{\text {tot }}$ is formally composed of five distinguishable integrals

$$
\delta \Psi_{\text {int }}^{\text {tot }}=\int_{\mathcal{B}_{0}} \delta \boldsymbol{\varphi} \cdot\{\bullet\} \mathrm{d} V+\int_{\mathcal{S}_{0}} \delta \boldsymbol{\varphi} \cdot\{\bullet\} \mathrm{d} A+\int_{\mathcal{I}_{0}} \delta \overline{\boldsymbol{\varphi}} \cdot\{\bullet\} \mathrm{d} A+\int_{\mathcal{I}_{0}} \delta \overline{\boldsymbol{f}} \cdot\{\bullet\} \mathrm{d} A+\int_{\mathcal{C}_{0}} \delta \overline{\boldsymbol{\varphi}} \cdot\{\bullet\} \mathrm{d} L .
$$

2.2.2. External energy. The total external energy functional $\Psi_{\text {ext }}^{\text {tot }}$ in its most general form can consist of the contributions from externally prescribed forces in the bulk $\mathcal{B}_{0}$ and also on the surface $\mathcal{S}_{0}$, interface $\mathcal{I}_{0}$ and curve $\mathcal{C}_{0}$. Without loss of generality, $\Psi_{\text {ext }}^{\text {tot }}$ reads

$$
\Psi_{\text {ext }}^{\text {tot }}=\int_{\mathcal{B}_{0}} \psi_{\text {ext }} \mathrm{d} V+\int_{\mathcal{S}_{0}} \widehat{\psi}_{\text {ext }} \mathrm{d} A+\int_{\mathcal{I}_{0}} \bar{\psi}_{\text {ext }} \mathrm{d} A+\int_{\mathcal{C}_{0}} \widetilde{\psi}_{\text {ext }} \mathrm{d} L
$$

in which $\psi_{\text {ext }}, \widehat{\psi}_{\text {ext }}, \bar{\psi}_{\text {ext }}$ and $\widetilde{\psi}_{\text {ext }}$ denote the external energy densities of the bulk $\mathcal{B}_{0}$, surface $\mathcal{S}_{0}$, interface $\mathcal{I}_{0}$ and curve $\mathcal{C}_{0}$, respectively. Since the variation of the total energy (3) must vanish, and motivated by the format of the variation of the total internal energy (22), the first variation of the total external energy functional $\delta \Psi_{\text {ext }}^{\text {tot }}$ should be of the form

$$
\delta \Psi_{\text {ext }}^{\text {tot }}=-\int_{\mathcal{B}_{0}} \delta \boldsymbol{\varphi} \cdot \boldsymbol{b}_{0} \mathrm{~d} V-\int_{\mathcal{S}_{0}} \delta \boldsymbol{\varphi} \cdot \widehat{\boldsymbol{b}}_{0} \mathrm{~d} A-\int_{\mathcal{I}_{0}} \delta \overline{\boldsymbol{\varphi}} \cdot \overline{\boldsymbol{b}}_{0} \mathrm{~d} A-\int_{\mathcal{I}_{0}} \delta \overline{\boldsymbol{f}} \cdot \overline{\boldsymbol{b}}_{0}^{*} \mathrm{~d} A-\int_{\mathcal{C}_{0}} \delta \overline{\boldsymbol{\varphi}} \cdot \widetilde{\boldsymbol{b}}_{0} \mathrm{~d} L .
$$

The term $\boldsymbol{b}_{0}$ denotes the body force density in the material configuration. Analogously, $\widehat{\boldsymbol{b}}_{0}$ denotes the surface force per unit area, often referred to as traction. The interface force densities are denoted $\overline{\boldsymbol{b}}_{0}$ and $\overline{\boldsymbol{b}}_{0}^{*}$ as the work (external energy) conjugates to the interface motion $\bar{\varphi}$ and the interface motion jump $\bar{f}$, respectively. The external force density along the curve per unit length in the material configuration is denoted $\widetilde{\boldsymbol{b}}_{0}$. 
2.2.3. Balance equations. In order to derive the balance equations, the total energy $\delta \Psi^{\text {tot }}$ and the first variation of the total energy $\delta \Psi^{\text {tot }}$ must vanish. Clearly, the variation of the total energy $\delta \Psi^{\text {tot }}$ corresponds to all admissible spatial variations of the motion $\delta \varphi$ and consequently $\delta \bar{\varphi}$ and $\delta \bar{f}$. Recall, both $\bar{\varphi}$ and $\bar{f}$ are functions of the motion $\boldsymbol{\varphi}$. Substituting the variations of the total internal energy (21) and the total external energy (24) into the variation of the total energy (3) furnishes the global balance equation

$$
\begin{gathered}
-\int_{\mathcal{B}_{0}} \delta \boldsymbol{\varphi} \cdot\left[\operatorname{Div} \boldsymbol{P}+\boldsymbol{b}_{0}\right] \mathrm{d} V+\int_{\mathcal{S}_{0}} \delta \boldsymbol{\varphi} \cdot\left[\boldsymbol{P} \cdot \widehat{\boldsymbol{N}}-\widehat{\boldsymbol{b}}_{0}\right] \mathrm{d} A-\int_{\mathcal{I}_{0}} \delta \overline{\boldsymbol{\varphi}} \cdot\left[\overline{\operatorname{Div}} \overline{\boldsymbol{P}}+\llbracket \boldsymbol{P} \rrbracket \cdot \overline{\boldsymbol{N}}+\overline{\boldsymbol{b}}_{0}\right] \mathrm{d} A \\
\quad+\int_{\mathcal{I}_{0}} \delta \overline{\boldsymbol{f}} \cdot\left[\overline{\boldsymbol{t}}-\{[\boldsymbol{P}\}\} \cdot \overline{\boldsymbol{N}}-\overline{\boldsymbol{b}}_{0}^{*}\right] \mathrm{d} A+\int_{\mathcal{C}_{0}} \delta \overline{\boldsymbol{\varphi}} \cdot\left[\overline{\boldsymbol{P}} \cdot \tilde{\boldsymbol{N}}-\widetilde{\boldsymbol{b}}_{0}\right] \mathrm{d} L=0,
\end{gathered}
$$

from which the local balance equations can be obtained due to arbitrariness of the spatial variations $\delta \bar{\varphi}$ as

$$
\begin{array}{ll}
\operatorname{Div} \boldsymbol{P}+\boldsymbol{b}_{0}=\mathbf{0} & \text { in } \mathcal{B}_{0}, \\
\boldsymbol{P} \cdot \widehat{\boldsymbol{N}}-\widehat{\boldsymbol{b}}_{0}=\mathbf{0} & \text { on } \mathcal{S}_{0}, \\
\overline{\operatorname{Div}} \overline{\boldsymbol{P}}+\llbracket \boldsymbol{P} \rrbracket \cdot \overline{\boldsymbol{N}}+\overline{\boldsymbol{b}}_{0}=\mathbf{0} & \text { on } \mathcal{I}_{0} \text { (along), } \\
\overline{\boldsymbol{t}}-\{\{\boldsymbol{P}\}\} \cdot \overline{\boldsymbol{N}}-\overline{\boldsymbol{b}}_{0}^{\circ}=\mathbf{0} & \text { on } \mathcal{I}_{0} \text { (across), } \\
\overline{\boldsymbol{P}} \cdot \tilde{\boldsymbol{N}}-\widetilde{\boldsymbol{b}}_{0}=\mathbf{0} & \text { on } \mathcal{C}_{0} .
\end{array}
$$

Note that the balance equation in the bulk is the well-established balance of linear momentum in a geometrically non-linear continuum mechanics setting. The balance equation on the surface exactly resembles the Cauchy relation between external traction and stress in the material. The generalized Young-Laplace equation can be identified as the balance equation along the interface. Appendix 2 provides details on how the balance equation along the interface $(26)_{3}$ reduces to the classical Young-Laplace equation. The relation between the interface traction and the average stress along the interface is the balance equation across the interface. The balance equation on the curve shall be understood as a Cauchy-type relation to link external traction on the curve and the stress on the interface.

In passing, it shall be noted that the interface force densities $\overline{\boldsymbol{b}}_{0}$ and $\overline{\boldsymbol{b}}_{0}^{\circ}$ for a purely mechanical problem seem irrelevant and impossible to be imposed externally. However, they are inseparable elements of the balance equations and are included for the sake of completeness. Furthermore, one can imagine certain scenarios in multi-physics where the interface force densities can be applied externally. For instance, imagine coated particles in a matrix where the coating is sensitive to external magnetic or electric fields inducing various reactions leading to a force density. In the absence of the interface force densities $\overline{\boldsymbol{b}}_{0}$ and $\overline{\boldsymbol{b}}_{0}^{\circ}$, the interface balance equations read

$$
\overline{\operatorname{Div}} \overline{\boldsymbol{P}}+\llbracket \boldsymbol{P} \rrbracket \cdot \overline{\boldsymbol{N}}=\mathbf{0} \quad \text { on } \mathcal{I}_{0} \text { (along) } \quad \text { and } \quad \overline{\boldsymbol{t}}-\{\{\boldsymbol{P}\}\} \cdot \overline{\boldsymbol{N}}=\mathbf{0} \quad \text { on } \mathcal{I}_{0} \text { (across). }
$$

Obviously, for vanishing elastic resistance along the interface, that is, $\overline{\boldsymbol{P}}=\mathbf{0}$, the interface balance equations simplify to

$$
\llbracket \boldsymbol{P} \rrbracket \cdot \overline{\boldsymbol{N}}=\mathbf{0} \quad \text { on } \mathcal{I}_{0} \text { (along) } \quad \text { and } \quad \overline{\boldsymbol{t}}=\{\{\boldsymbol{P}\}\} \cdot \overline{\boldsymbol{N}} \quad \text { on } \mathcal{I}_{0} \text { (across), }
$$

which are the well-established governing equations of the cohesive interface model. In particular, $\llbracket \boldsymbol{P} \rrbracket \cdot \overline{\boldsymbol{N}}=\mathbf{0}$ states explicitly that the traction jump across the interface must vanish.

\subsection{Material modeling}

In order to proceed, the internal free energy densities $\psi_{\text {int }}$ and $\bar{\psi}_{\text {int }}$ for the bulk and interface respectively shall be specified. Constitutive responses of the bulk and interface are then derived through the definitions

$$
\boldsymbol{P}:=\frac{\partial \psi_{\text {int }}}{\partial \boldsymbol{F}} \quad \text { in } \mathcal{B}_{0} \quad \text { and } \quad \overline{\boldsymbol{P}}:=\frac{\partial \bar{\psi}_{\text {int }}}{\partial \overline{\boldsymbol{F}}}, \quad \overline{\boldsymbol{t}}:=\frac{\partial \bar{\psi}_{\text {int }}}{\partial \overline{\boldsymbol{f}}} \quad \text { on } \mathcal{I}_{0} .
$$

For the material response of the bulk, an isotropic hyperelastic neo-Hookean energy density in the material configuration

$$
\psi_{\text {int }}(\boldsymbol{F})=\frac{1}{2} \mu\left[J^{-2 / 3} \boldsymbol{F}: \boldsymbol{F}-3\right]+\kappa\left[\frac{1}{4} J^{2}-\frac{1}{2} \ln J-\frac{1}{4}\right] \quad \text { with } \quad J:=\operatorname{Det} \boldsymbol{F}
$$


is chosen with $\mu$ being the (first) Lamé constant and $\kappa$, the bulk modulus. This choice for the bulk energy density is suitable for rubber-like materials and benefits from the original ideas of Ogden [94] to capture the macroscopic behavior of nearly incompressible soft polymers. In general, it is possible to develop physically motivated material models through fundamental reasoning. Material modeling is a mature field and further discussions on the choice of the bulk material is outside the scope of this manuscript. From a variational perspective, it is of crucial importance that the energy density $\psi_{\text {int }}$ satisfies the material frame indifference in the sense that

$$
\psi_{\text {int }}(\boldsymbol{F})=\psi_{\text {int }}(\boldsymbol{Q} \cdot \boldsymbol{F}) \quad \forall \boldsymbol{Q} \in \mathcal{S O}(3),
$$

in which $\boldsymbol{Q}$ denotes an arbitrary proper orthogonal tensor with the properties $\boldsymbol{Q}^{\mathrm{t}}=\boldsymbol{Q}^{-1}$ and Det $\boldsymbol{Q}=1$. It can be readily shown (see e.g. [78]) that the energy density $\psi_{\text {int }}$ is frame-indifferent if the deformation gradient $\boldsymbol{F}$ enters the energy through the right Cauchy-Green tensor $\boldsymbol{C}$ as

$$
\psi=\psi(\boldsymbol{F}) \quad===\stackrel{\text { material frame indifference }}{=}==\Longrightarrow \quad \psi=\psi(\boldsymbol{C}) \text { with } \quad \boldsymbol{C}:=\boldsymbol{F}^{\mathrm{t}} \cdot \boldsymbol{F} .
$$

The bulk energy density (30) is indeed frame-indifferent since it can be expressed in terms of $\boldsymbol{C}$ instead of $\boldsymbol{F}$ as

$$
\psi_{\text {int }}(\boldsymbol{C})=\frac{1}{2} \mu\left[J^{-2 / 3} \boldsymbol{C}: \boldsymbol{I}-3\right]+\kappa\left[\frac{1}{4} J^{2}-\frac{1}{2} \ln J-\frac{1}{4}\right] \quad \text { with } \quad J:=\sqrt{\operatorname{Det} \boldsymbol{C}} .
$$

For the given bulk energy density (30), the Piola stress $\boldsymbol{P}$ reads

$$
\boldsymbol{P}:=\frac{\partial \psi_{\mathrm{int}}}{\partial \boldsymbol{F}}=\mu J^{-2 / 3}\left[\boldsymbol{F}-\frac{1}{3} \boldsymbol{F}: \boldsymbol{F}^{-\mathrm{t}}\right]+\frac{1}{2} \kappa\left[J^{2}-1\right] \boldsymbol{F}^{\mathrm{t}} .
$$

For the material response of the interface, the interface energy density $\bar{\psi}_{\text {int }}=\bar{\psi}_{\text {int }}(\overline{\boldsymbol{F}}, \overline{\boldsymbol{f}})$ is additively decomposed into its portion along the interface $\bar{\psi}_{\text {int }}^{\|}$and the portion across the interface $\bar{\psi}_{\text {int }}^{\perp}$ as

$$
\bar{\psi}_{\text {int }}(\overline{\boldsymbol{F}}, \overline{\boldsymbol{f}})=\bar{\psi}_{\text {int }}^{\|}(\overline{\boldsymbol{F}})+\bar{\psi}_{\text {int }}^{\perp}(\overline{\boldsymbol{f}}) \quad \text { with } \quad \overline{\boldsymbol{F}}:=\overline{\operatorname{Grad}}\{\{\boldsymbol{\varphi}\}\}, \quad \overline{\boldsymbol{f}}:=\llbracket \boldsymbol{\varphi} \rrbracket .
$$

Clearly, this choice is made for convenience and motivated by the fact that the general interface model here should recover both elastic interface and cohesive interface models in its limits. Similar to the bulk, the interface energy density $\bar{\psi}_{\text {int }}$ must satisfy the material frame indifference in the sense that

$$
\bar{\psi}_{\text {int }}(\overline{\boldsymbol{F}}, \overline{\boldsymbol{f}})=\bar{\psi}_{\mathrm{int}}(\boldsymbol{Q} \cdot \overline{\boldsymbol{F}}, \boldsymbol{Q} \cdot \overline{\boldsymbol{f}}) \quad \forall \boldsymbol{Q} \in \mathcal{S O}(3) .
$$

The interface energy density $\bar{\psi}_{\text {int }}$ is frame-indifferent if and only if it can be represented as

$$
\bar{\psi}_{\mathrm{int}}(\overline{\boldsymbol{C}}, \bar{c})=\bar{\psi}_{\mathrm{int}}^{\|}(\overline{\boldsymbol{C}})+\bar{\psi}_{\mathrm{int}}^{\perp}(\bar{c}) \quad \text { with } \quad \overline{\boldsymbol{C}}:=\overline{\boldsymbol{F}}^{\mathrm{t}} \cdot \overline{\boldsymbol{F}}, \quad \bar{c}:=\overline{\boldsymbol{f}} \cdot \overline{\boldsymbol{f}},
$$

in which $\overline{\boldsymbol{C}}$ denotes the interface right Cauchy-Green tensor and $\bar{c}$ is the square of the norm of the displacement jump across the interface.

A basic model to capture the behavior of the interface is the isotropic interface model for the in-plane response $\bar{\psi}_{\text {int }}^{\|}$and a cohesive-type model for the orthogonal response $\bar{\psi}_{\text {int }}^{\perp}$. A physically sound interface model must (i) satisfy the invariance with respect to rigid body motions and (ii) fulfill certain (poly)convexity conditions. Following the representation theorem for isotropic functions $[95,96]$ and in compliance with equation (37), the interface energy $\bar{\psi}_{\text {int }}$ is chosen to be a function of invariants of $\overline{\boldsymbol{C}}$ and $\bar{c}$. Clearly, the interface right Cauchy-Green tensor $\overline{\boldsymbol{C}}$ captures the in-plane response of the interface while $\bar{c}$ recovers the orthogonal response. Thus, the interface behavior is expressed in the general form

$$
\bar{\psi}_{\text {int }}=\bar{\psi}_{\text {int }}\left(\bar{I}_{1}, \bar{I}_{2}, \bar{I}_{3}\right) \quad \text { with } \quad \bar{I}_{1}:=\overline{\boldsymbol{C}}: \overline{\boldsymbol{I}}, \quad \bar{I}_{2}:=\overline{\operatorname{Det}} \overline{\boldsymbol{C}}, \quad \bar{I}_{3}:=\sqrt{\bar{c}}
$$

where $\bar{I}_{1}, \bar{I}_{2}$ and $\bar{I}_{3}$ are the invariants of $\overline{\boldsymbol{C}}$ and $\bar{c}$, respectively. An interesting consequence of the isotropic interface response is that the interface stress and traction, without loss of generality, simplify to

$$
\overline{\boldsymbol{P}}:=\frac{\partial \bar{\psi}_{\text {int }}}{\partial \overline{\boldsymbol{F}}}=2 \frac{\partial \bar{\psi}_{\text {int }}}{\partial \bar{I}_{1}} \overline{\boldsymbol{F}}+2 \frac{\partial \bar{\psi}_{\text {int }}}{\partial \bar{I}_{2}} \bar{I}_{2} \overline{\boldsymbol{F}}^{\text {-t }} \quad \text { and } \quad \overline{\boldsymbol{t}}:=\frac{\partial \bar{\psi}_{\text {int }}}{\partial \overline{\boldsymbol{f}}}=\frac{1}{\bar{I}_{3}} \frac{\partial \bar{\psi}_{\text {int }}}{\partial \bar{I}_{3}} \overline{\boldsymbol{f}} .
$$


Next, the isotropic frame-indifferent interface energy (38) is decomposed into its in-plane and orthogonal contributions $\bar{\psi}_{\text {int }}^{\|}$and $\bar{\psi}_{\text {int }}^{\perp}$, respectively, as

$$
\bar{\psi}_{\text {int }}(\overline{\boldsymbol{F}}, \overline{\boldsymbol{f}})=\bar{\psi}_{\text {int }}^{\|}(\overline{\boldsymbol{F}})+\bar{\psi}_{\text {int }}^{\perp}(\overline{\boldsymbol{f}})===\stackrel{\text { material frame indifference }+ \text { isotropy }}{=}=\stackrel{=}{=}==
$$

In order to a priori satisfy the (poly)convexity conditions for the interface and to fulfill the corresponding growth conditions on the interface, the specific interface energy density

$$
\bar{\psi}_{\text {int }}\left(\bar{I}_{1}, \bar{I}_{2}, \bar{I}_{3}\right)=\frac{1}{2} \bar{\mu}\left[\bar{I}_{1}-2-2 \ln \sqrt{\bar{I}_{2}}\right]+\frac{1}{2} \bar{\lambda}\left[\frac{1}{2}\left[\bar{I}_{2}-1\right]-\ln \sqrt{\bar{I}_{2}}\right]+\frac{1}{2} \bar{\alpha}_{3}^{2}
$$

is chosen in which $\bar{\mu}, \bar{\lambda}$ and $\bar{\alpha}$ are the interface material parameters. The interface energy density (41) can be written as a function of the interface deformation gradient $\overline{\boldsymbol{F}}$ and the interface displacement jump $\overline{\boldsymbol{f}}$ as

$$
\bar{\psi}_{\text {int }}(\overline{\boldsymbol{F}}, \overline{\boldsymbol{f}})=\frac{1}{2} \bar{\mu}[\overline{\boldsymbol{F}}: \overline{\boldsymbol{F}}-2-2 \ln \bar{J}]+\frac{1}{2} \bar{\lambda}\left[\frac{1}{2}\left[\bar{J}^{2}-1\right]-\ln \bar{J}\right]+\frac{1}{2} \bar{\alpha} \overline{\boldsymbol{f}} \cdot \overline{\boldsymbol{f}} \quad \text { with } \quad \bar{J}=\overline{\operatorname{Det}} \overline{\boldsymbol{F}} .
$$

For the given interface energy density (42), the interface stress $\overline{\boldsymbol{P}}$ and the interface traction $\overline{\boldsymbol{t}}$ read

$$
\overline{\boldsymbol{P}}:=\frac{\partial \bar{\psi}_{\mathrm{int}}}{\partial \overline{\boldsymbol{F}}}=\bar{\mu}\left[\overline{\boldsymbol{F}}-\overline{\boldsymbol{F}}^{-\mathrm{t}}\right]+\frac{1}{2} \bar{\lambda}\left[\bar{J}^{2}-1\right] \overline{\boldsymbol{F}}^{-\mathrm{t}} \quad \text { and } \quad \overline{\boldsymbol{t}}:=\frac{\partial \bar{\psi}_{\mathrm{int}}}{\partial \overline{\boldsymbol{f}}}=\bar{\alpha} \overline{\boldsymbol{f}}
$$

The interface material parameters $\bar{\mu}, \bar{\lambda}$ and $\bar{\alpha}$ describe distinctive behavior of an interface. Both $\bar{\mu}$ and $\bar{\lambda}$ correspond to the in-plane interface response while $\bar{\alpha}$ determines the orthogonal stiffness of an interface. The in-plane parameters $\bar{\mu}$ and $\bar{\lambda}$ have the unit $\mathrm{N} / \mathrm{m}$ and shall be understood as interface Lamé parameters. The orthogonal stiffness $\bar{\alpha}$ with the unit $\mathrm{N} / \mathrm{m}^{3}$ indicates the resistance of the interface to opening and shall be understood as the isotropic cohesive parameter. In the limit of vanishing $\bar{\alpha}$, the general interface model exhibits no resistance to opening. Increasing $\bar{\alpha}$ strengthens the interface resistance to opening. In the limit of $\bar{\alpha} \rightarrow \infty$, the interface opening ultimately vanishes and, thus, the general interface model behaves in a geometrically coherent manner. The numerical examples in the next section are devised to clearly illustrate the role of the interface parameters. The computational aspects of the general interface model using the finite element method with application to homogenization are discussed in [97, 98].

\section{Illustration of the theory}

The main goal of this section is to elucidate the nature of interface parameters via numerical examples. The numerical examples are limited to two-dimensional illustrations corresponding to plane strain for the sake of simplicity. Consider the unit square shown in Figure 6. The domain is partitioned into two disjoint subdomains $\mathcal{B}_{0}^{-}$and $\mathcal{B}_{0}^{+}$by the interface $\mathcal{I}_{0}$. The specimen is stretched by applying prescribed displacements on its edges. Lateral deformations are prevented and, hence, the width of the specimen cannot change. The example is devised such that it clearly demonstrates both in-plane and orthogonal interface responses. For the given boundary conditions, a straight interface could only capture either the in-plane or the orthogonal response. Thus, the interface here is assumed to be curved.

The material parameters are chosen to amplify the impact of interfaces such that the influences of different interface models are distinctly noticeable. The purpose of the examples here is to better describe the general interface model and the essence of its parameters without introducing too much complexity. That is, the examples here deliver only parametric studies and are purely computational. To link such numerical observations to the physics of materials, one certainly needs more information about the micro-structure itself and its constitutive response. For the two-dimensional problem of interest here, both in-plane parameters $\bar{\mu}$ and $\bar{\lambda}$ serve the same purpose and resist the change of length of the interface. Therefore, without loss of generality, the interface parameter $\bar{\lambda}$ is set to zero and $\bar{\mu}$ remains the only parameter to capture the elastic resistance along the interface. The material parameters are chosen as $\mu=10 \mathrm{~N} / \mathrm{mm}^{2}, \kappa=50 \mathrm{~N} / \mathrm{mm}^{2}, \bar{\mu}=100 \mathrm{~N} / \mathrm{mm}$ and $\bar{\alpha}=100 \mathrm{~N} / \mathrm{mm}^{3}$. Of course, the numerical observations here hold qualitatively for any set of material parameters. 

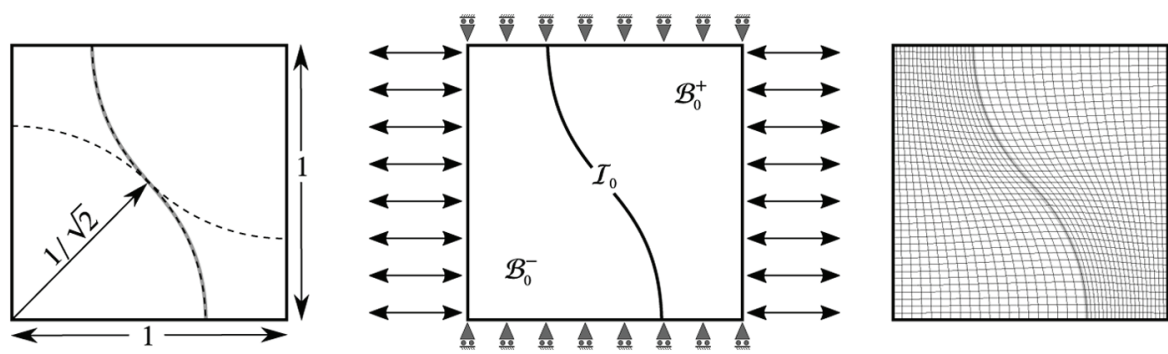

Figure 6. Unit square with interface. Geometry (left), boundary conditions (center) and discretization (right). The domain is extended via prescribed displacements at the edges. Lateral deformations are prevented in the sense that the width of the domain cannot vary throughout the deformation process.
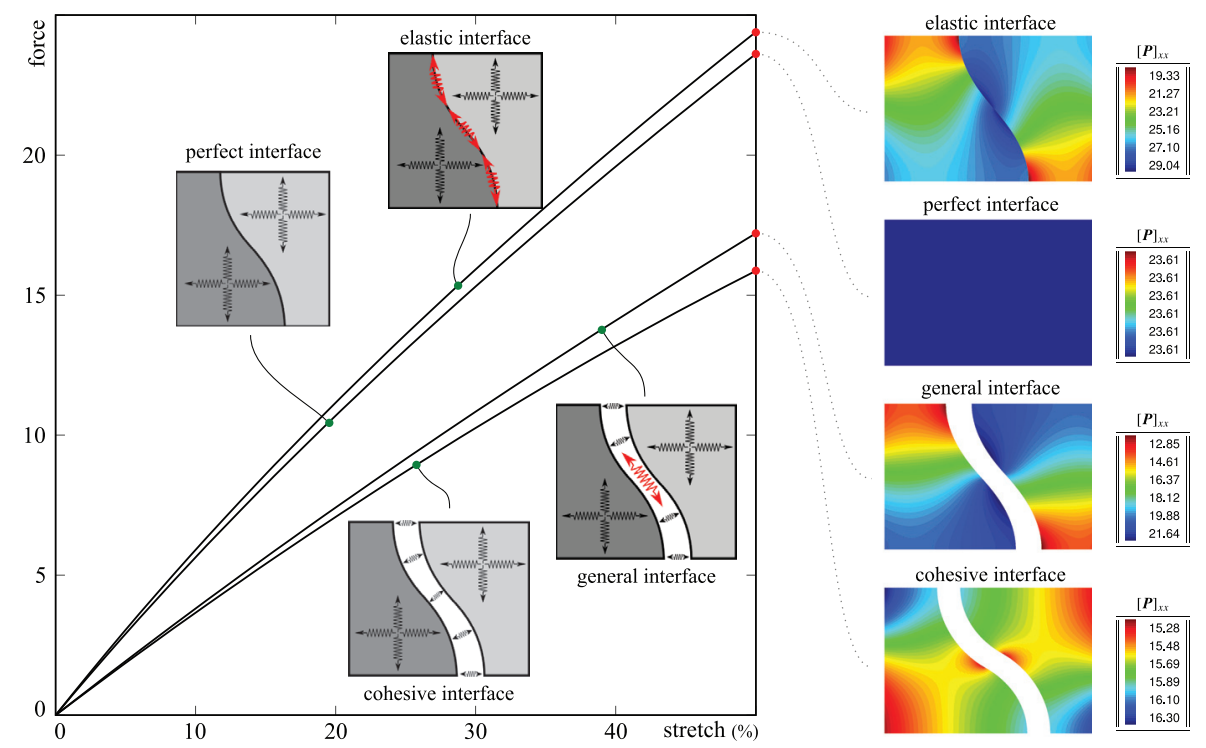

Figure 7. Illustration of the influence of various interface types on the overall material response. The graph shows the total force on the edges versus the prescribed stretch on the specimen. The results on the right display the Piola stress distribution within the bulk at $50 \%$ stretch. It can be clearly seen that the traction jump across the interface vanishes for both perfect and cohesive interface models, but not across the elastic and general interfaces.

Figure 7 gathers the results of several numerical simulations corresponding to different types of interface behavior. The graph shows the overall force required to stretch the specimen versus stretch. Neither a perfect nor an elastic interface allows for the jump of the displacement across the interface and, hence, there is no opening. The perfect interface model does not cause a traction jump across the interface and, therefore, a uniform stress pattern is observed. The elastic interface model shows in-plane resistance along the interface. Due to the curved topology of the interface, the stress along the interface leads to a stress jump across the interface. Furthermore, due to additional resistance along the interface, the overall force required to apply the stretch is higher than that for the perfect interface model. The cohesive interface model allows for the displacement jump across the interface, but has no in-plane resistance along the interface. As a consequence, the stress distribution is not uniform for this model and, overall, less force is required on the edges to prescribe the same stretch compared to other interface types. Note that the normal stress jump across the interface vanishes for the cohesive interface model. The general interface model allows for opening, but also shows resistance along the interface leading to a rather complex overall response. The stress jump across the interface is clearly noticeable for the general interface model. The next example clarifies the role of the interface material parameters in the general interface model.

In order to better understand the influence of the interface material parameters, consider Figure 6 where the interface type is general imperfect and 50\% stretch is prescribed on the domain. Obviously, for vanishing $\bar{\mu}$ the interface shows no in-plane resistance at all. The orthogonal stiffness $\bar{\alpha}$ varies from zero to infinity. At the limit 


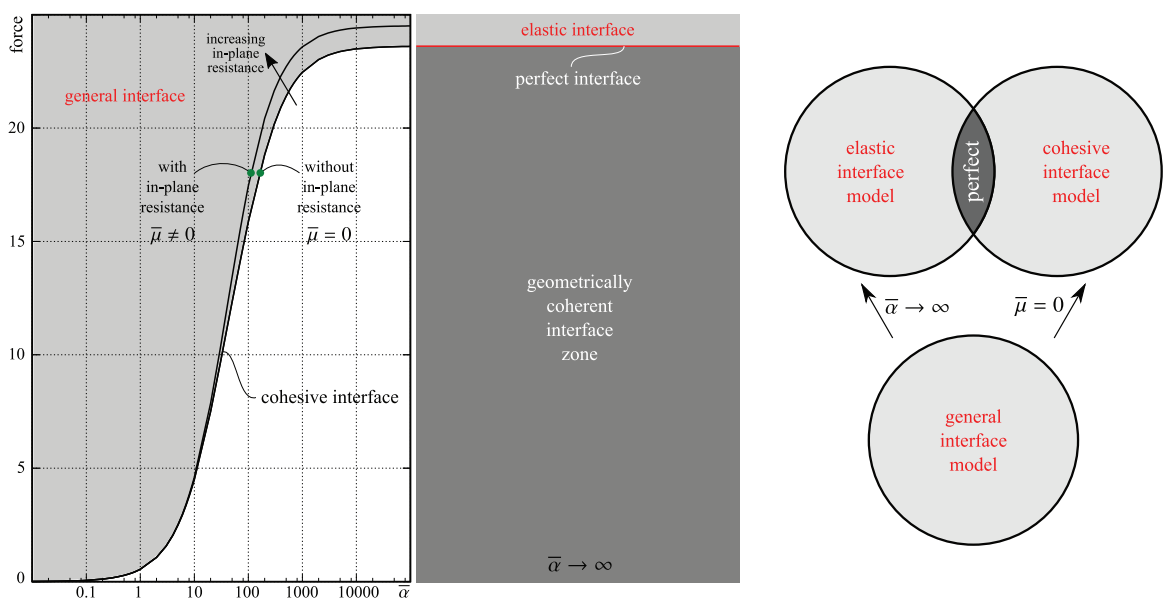

Figure 8. Illustration of the influence of the interface material parameters. A general interface model coincides with the cohesive interface model in the absence of the interface in-plane resistance. In the limit of infinitely large orthogonal resistance, the general interface model converges to the elastic interface model. The perfect interface model can be understood as the intersection of cohesive and elastic interface models.

of $\bar{\alpha}=0$, the prescribed deformations require no force on the edges. Increasing $\bar{\alpha}$ leads to larger forces on the edges. In the limit of $\bar{\alpha} \rightarrow \infty$ the interface opening vanishes and the general interface model asymptotically converges to the elastic interface model; see Figure 8. The elastic interface model with $\bar{\mu}=0$ matches precisely with the perfect interface model though and, thus, the cohesive interface model at the limit of $\bar{\alpha} \rightarrow \infty$ converges to the perfect interface model.

\section{Summary}

Commonly accepted strategies to capture interface behavior fall into the two categories of elastic or cohesive interfaces. It is shown that both the elastic and cohesive interface models can be unified as the limit cases of a broader model, namely the general interface model. The general interface model at finite deformation elasticity is formulated in a variationally consistent manner. Typical applications of the model include nano-materials due to the increasing area-to-volume ratio at smaller dimensions. In summary, this manuscript presents an attempt to shed light on generalized interfaces. This generic framework is broadly applicable to improving the understanding of the size-dependent behavior of continua with a large variety of applications in nano-materials and polycrystalline solids.

\section{Funding}

The author(s) received no financial support for the research, authorship, and/or publication of this article.

\section{Notes}

1. Obviously, it is possible to define the interface motion $\bar{\varphi}$ differently from the motion of the mid-surface. A different choice would require more complicated notation without providing any further insight. Strictly speaking, any other choice for the interface motion would result in governing equations slightly different from the ones in this contribution. Nevertheless, the procedure to derive the governing equations is formally identical to what is carried out in Section 2.2. Without having more information about the specific interphase of interest, any other choice is equally justifiable.

2. The term "Piola stress" is adopted instead of the more commonly used "first Piola-Kirchhoff stress". Nonetheless, it seems that Piola stress is a more appropriate choice for this stress measure. Recall, $\boldsymbol{P}$ is essentially the Piola transform of the Cauchy stress and ties perfectly to the Piola identity. Also, historically, Kirchhoff (1824-1877) employed this stress measure after Piola (1794-1850); see also the discussion in [92]. For further details on the works of Piola, see [93] and the references therein.

3. There are various conventions to define the mean curvature in the literature. For instance, in [78] the term "mean curvature" refers to the sum of the principal curvatures or the trace of the curvature tensor. Here, another more intuitive definition of the mean curvature is adopted as the arithmetic mean of the principal curvatures and, thus, $\bar{K}$ denotes twice the mean curvature. 


\section{References}

[1] Hill, R. Elastic properties of reinforced solids: Some theoretical principles. J Mech Phys Solid 1963; 11(5): 357-372.

[2] Hill, R. On constitutive macro-variables for heterogeneous solids at finite strain. Proc R Soc A Math Phys Eng Sci 1972; 326(1565): 131-147.

[3] Ogden, R. On the overall moduli of non-linear elastic composite materials. J Mech Phys Solid 1974; 22(6): $541-553$.

[4] Kanouté, P, Boso, DP, Chaboche, JL, et al. Multiscale methods for composites: A review. Arch Comput Meth Eng 2009; 16(1): 31-75.

[5] Geers, MGD, Kouznetsova, VG, and Brekelmans, WAM. Multi-scale computational homogenization: Trends and challenges. $J$ Comput Appl Mech 2010; 234(7): 2175-2182.

[6] Ostoja-Starzewski, M, Kale, S, Karimi, P, et al. Scaling to RVE in random media. Adv Appl Mech 2016; 49: $111-211$.

[7] Saeb, S, Steinmann, P and Javili, A. Aspects of computational homogenization at finite deformations: A unifying review from Reuss' to Voigt's bound. Appl Mech Rev 2016; 68: 050801.

[8] Matous, K, Geers, MG, Kouznetsova, VG, et al. A review of predictive nonlinear theories for multiscale modeling of heterogeneous materials. J Comput Phys 2017; 330: 192-220.

[9] Hashin, Z. Thin interphase/imperfect interface in elasticity with application to coated fiber composites. J Mech Phys Solid 2002; 50: 2509-2537.

[10] McBride, A, Mergheim, J, Javili, A, et al. Micro-to-macro transitions for heterogeneous material layers accounting for in-plane stretch. J Mech Phys Solid 2012; 60(6): 1221-1239.

[11] Daher, N and Maugin, GA. The method of virtual power in continuum mechanics application to media presenting singular surfaces and interfaces. Acta Mech 1986; 60(3-4): 217-240.

[12] Chen, T, Chiu, MS, and Weng, CN. Derivation of the generalized Young-Laplace equation of curved interfaces in nanoscaled solids. J Appl Phys 2006; 100(7): 074308.

[13] Javili, A, McBride, A, and Steinmann, P. Thermomechanics of solids with lower-dimensional energetics: On the importance of surface, interface, and curve structures at the nanoscale. A unifying review. Appl Mech Rev 2013; 65(1): 010802.

[14] Moeckel, GP. Thermodynamics of an interface. Arch Rat Mech Anal 1975; 57(3): 255-280.

[15] Murdoch, AI. A thermodynamical theory of elastic material interfaces. QJ Mech Appl Math 1976; 29(3): $245-275$.

[16] Dell'Isola, F, and Romano, A. On the derivation of thermomechanical balance equations for continuous systems with a nonmaterial interface. Int J Eng Sci 1987; 25(11-12): 1459-1468.

[17] Fried, E, and Gurtin, ME. Thermomechanics of the interface between a body and its environment. Contin Mech Thermodyn 2007; 19(5): 253-271.

[18] Gurtin ME, and Murdoch AI. A continuum theory of elastic material surfaces. Arch Rat Mech Anal 1975; 57(4): $291-323$.

[19] Gurtin, ME, Weissmüller, J, and Larche, F. A general theory of curved deformable interfaces in solids at equilibrium. Philos Mag A 1998; 78(5): 1093-1109.

[20] Steigmann, DJ, and Ogden, RW. Elastic surface-substrate interactions. Proc R Soc A Math Phys Eng Sci 1999; 455(1982): 437-474.

[21] Fried, E, and Todres, RE. Mind the gap: The shape of the free surface of a rubber-like material in proximity to a rigid contactor. J Elast 2005; 80(1-3): 97-151.

[22] Huang, ZP, and Wang, J. A theory of hyperelasticity of multi-phase media with surface/interface energy effect. Acta Mech 2006; 182(3-4): 195-210.

[23] Steinmann, P. On boundary potential energies in deformational and configurational mechanics. J Mech Phys Solid 2008; 56(3): 772-800.

[24] Dingreville, R, and Qu, J. Interfacial excess energy, excess stress and excess strain in elastic solids: Planar interfaces. $J$ Mech Phys Solid 2008; 56(5): 1944-1954.

[25] Duan, HL, Wang, J, and Karihaloo, BL. Theory of elasticity at the nanoscale. Adv Appl Mech 2009; 42: 1-68.

[26] Wang, Y, Weissmüller, J, and Duan, HL. Mechanics of corrugated surfaces. J Mech Phys Solid 2010; 58(10): 1552-1566.

[27] Wang, ZQ, Zhao, YP, and Huang, ZP. The effects of surface tension on the elastic properties of nano structures. Int J Eng Sci 2010; 48(2): 140-150.

[28] Altenbach, H, and Eremeyev, VA. On the shell theory on the nanoscale with surface stresses. Int J Eng Sci 2011; 49(12): 1294-1301.

[29] Chhapadia, P, Mohammadi, P, and Sharma, P. Curvature-dependent surface energy and implications for nanostructures. $J$ Mech Phys Solid 2011; 59(10): 2103-2115.

[30] Zemlyanova, AY. The effect of a curvature-dependent surface tension on the singularities at the tips of a straight interface crack. Q J Mech Appl Math 2013; 66(2): 199-219.

[31] Dingreville, R, Hallil, A, and Berbenni, S. From coherent to incoherent mismatched interfaces: A generalized continuum formulation of surface stresses. J Mech Phys Solid 2014; 72(1): 40-60.

[32] Gao, X, Huang, Z, Qu, J, et al. A curvature-dependent interfacial energy-based interface stress theory and its applications to nano-structured materials: (I) General theory. J Mech Phys Solid 2014; 66(1): 59-77.

[33] Cordero, NM, Forest, S, and Busso, EP. Second strain gradient elasticity of nano-objects. J Mech Phys Solid 2016; 97 : $92-124$. 
[34] Liu, L, Yu, M, Lin, H, et al. Deformation and relaxation of an incompressible viscoelastic body with surface viscoelasticity. J Mech Phys Solid 2017; 98: 309-329.

[35] Barenblatt, GI. The formation of equilibrium cracks during brittle fracture. General ideas and hypotheses. Axially-symmetric cracks. J Appl Math Mech 1959; 23(3): 622-636.

[36] Barenblatt, GI. The mathematical theory of equilibrium cracks in brittle fracture. Adv Appl Mech 1962; 7: 55-129.

[37] Dugdale, D. Yielding of steel sheets containing slits. J Mech Phys Solid 1960; 8(2): 100-104.

[38] Needleman, A. A continuum model for void nucleation by inclusion debonding. J Appl Mech 1987; 54: 525-531.

[39] Xu, XP, and Needleman, A. Numerical simulations of fast crack growth in brittle solids. J Mech Phys Solid 1994; 42(9): 13971434.

[40] Ortiz, M, and Pandolfi, A. Finite-deformation irreversible cohesive elements for three-dimensional crack-propagation analysis. Int J Numer Meth Eng 1999; 44: 1267-1282.

[41] Tijssens, MGA, Sluys, BLJ, and Van der Giessen, E. Numerical simulation of quasi-brittle fracture using damaging cohesive surfaces. Eur J Mech A Solid 2000; 19(5): 761-779.

[42] Alfano, G, and Crisfield, MA. Finite element interface models for the delamination analysis of laminated composites: Mechanical and computational issues. Int J Numer Meth Eng 2001; 50: 1701-1736.

[43] Gasser, TC, and Holzapfel, GA. Geometrically non-linear and consistently linearized embedded strong discontinuity models for 3D problems with an application to the dissection analysis of soft biological tissues. Comput Meth Appl Mech Eng 2003; 192(47-48): 5059-5098.

[44] Van den Boschl, MJ, Schreurs, PJG, and Geers, MGD. An improved description of the exponential Xu and Needleman cohesive zone law for mixed-mode decohesion. Eng Fract Mech 2006; 73(9): 1220-1234.

[45] Fagerström, M, and Larsson, R. Theory and numerics for finite deformation fracture modelling using strong discontinuities. Int J Numer Meth Eng 2006; 66(6): 911-948.

[46] Charlotte, M, Laverne, J, and Marigo, JJ. Initiation of cracks with cohesive force models: A variational approach. Eur J Mech A Solid 2006; 25(4): 649-669.

[47] Park, K, Paulino, GH, and Roesler, JR. A unified potential-based cohesive model of mixed-mode fracture. J Mech Phys Solid 2009; 57(6): 891-908.

[48] Mosler, J, and Scheider, I. A thermodynamically and variationally consistent class of damage-type cohesive models. J Mech Phys Solid 2011; 59(8): 1647-1668.

[49] Park, K, and Paulino, GH. Cohesive zone models: A critical review of traction-separation relationships across fracture surfaces. Appl Mech Rev 2013; 64(6): 060802

[50] Dimitri, R, Trullo, M, De Lorenzis, L, et al. Coupled cohesive zone models for mixed-mode fracture: A comparative study. Eng Fract Mech 2015; 148: 145-179.

[51] Wu, C, Gowrishankar, S, Huang, R, et al. On determining mixed-mode traction-separation relations for interfaces. Int J Fract 2016; 202(1): 1-19.

[52] Qian, J, Lin, J, Xu, GK, et al. Thermally assisted peeling of an elastic strip in adhesion with a substrate via molecular bonds. J Mech Phys Solid 2017; 101: 197-208.

[53] Bövik, P. On the modelling of thin interface layers in elastic and acoustic scattering problems. QJ Mech Appl Math 1994; 47(1): $17-42$

[54] Benveniste, Y, and Miloh, T. Imperfect soft and stiff interfaces in two-dimensional elasticity. Mech Mater 2001; 33(6): 309-323.

[55] Benveniste, Y. A general interface model for a three-dimensional curved thin anisotropic interphase between two anisotropic media. J Mech Phys Solid 2006; 54(4): 708-734.

[56] Monchiet, V, and Bonnet, G. Interfacial models in viscoplastic composites materials. Int J Eng Sci 2010; 48(12): 1762-1768.

[57] Benveniste, Y, and Milton, GW. The effective medium and the average field approximations vis-a-vis the Hashin-Shtrikman bounds. I. The self-consistent scheme in matrix-based composites. J Mech Phys Solid 2010; 58(7): 1026-1038.

[58] Benveniste, Y, and Milton, GW. The effective medium and the average field approximations vis-a-vis the Hashin-Shtrikman bounds. II. The generalized self-consistent scheme in matrix-based composites. J Mech Phys Solid 2010; 58(7): 1039-1056.

[59] Benveniste, Y. Models of thin interphases with variable moduli in plane-strain elasticity. Math Mech Solid 2013; 18(2): 119-134.

[60] Gu, ST, Monteiro, E and He, QC. Coordinate-free derivation and weak formulation of a general imperfect interface model for thermal conduction in composites. Compos Sci Tech 2011; 71(9): 1209-1216.

[61] $\mathrm{Gu}, \mathrm{ST}$, and $\mathrm{He}, \mathrm{QC}$. Interfacial discontinuity relations for coupled multifield phenomena and their application to the modeling of thin interphases as imperfect interfaces. J Mech Phys Solid 2011; 59(7): 1413-1426.

[62] Matous, K, Kulkarni, MG, and Geubelle PH. Multiscale cohesive failure modeling of heterogeneous adhesives. $J$ Mech Phys Solid 2008; 56(4): 1511-1533.

[63] Kulkarni, MG, Geubelle, PH, and Matous, K. Multi-scale modeling of heterogeneous adhesives: Effect of particle decohesion. Mech Mater 2009; 41(5): 573-583.

[64] Kulkarni, MG, Matous, K, and Geubelle, PH. Coupled multi-scale cohesive modeling of failure in heterogeneous adhesives. Int J Numer Meth Eng 2010; 84: 916-946.

[65] Verhoosel, CV, Remmers, JJC, Gutiérrez, MA, et al. Computational homogenization for adhesive and cohesive failure in quasibrittle solids. Int J Numer Meth Eng 2010; 83: 1155-1179. 
[66] Cid Alfaro, MV, Suiker, ASJ, Verhoosel, CV et al. Numerical homogenization of cracking processes in thin fibre-epoxy layers. Eur J Mech A Solid 2010; 29(2): 119-131.

[67] Nguyen, VP, Lloberas-Valls O, Stroeven, M, et al. Homogenization-based multiscale crack modelling: From micro-diffusive damage to macro-cracks. Comput Meth Appl Mech Eng 2011; 200(9-12): 1220-1236.

[68] Levitas, VI and Warren, JA. Phase field approach with anisotropic interface energy and interface stresses: Large strain formulation. J Mech Phys Solid 2016; 91: 94-125.

[69] Petryk, H, and Stupkiewicz, S. Interfacial energy and dissipation in martensitic phase transformations. Part I: Theory. $J$ Mech Phys Solid 2010; 58(3): 390-408.

[70] Petryk, H, Stupkiewicz, S and Maciejewski G. Interfacial energy and dissipation in martensitic phase transformations. Part II: Size effects in pseudoelasticity. J Mech Phys Solid 2010; 58(3): 373-389.

[71] Tuma K, Stupkiewicz, S, and Petryk H. Size effects in martensitic microstructures: Finite-strain phase field model versus sharpinterface approach. J Mech Phys Solid 2016; 95: 284-307.

[72] Simha, NK, and Bhattacharya, K. Kinetics of phase boundaries with edges and junctions. J Mech Phys Solid 1998; 46(12): 2323-2359.

[73] Simha, NK and Bhattacharya, K. Kinetics of phase boundaries with edges and junctions in a three-dimensional multi-phase body. J Mech Phys Solid 2000; 48(12): 2619-2641.

[74] Spring, DW, Giraldo-Londoño, O, and Paulino, GH. A study on the thermodynamic consistency of the Park-Paulino-Roesler (PPR) cohesive fracture model. Mech Res Commun 2016; 78: 1-23.

[75] Steinmann, P, and Häsner O. On material interfaces in thermomechanical solids. Arch Appl Mech 2005; 75(1): 31-41.

[76] Esmaeili, A, Steinmann, P, and Javili, A. Non-coherent energetic interfaces accounting for degradation. Comput Mech 2017; 59: 361-383.

[77] Davydov, D, Javili, A, and Steinmann, P. On molecular statics and surface-enhanced continuum modeling of nano-structures. Comput Mater Sci 2013; 69: 510-519.

[78] Marsden, JE, and Hughes, TJR. Mathematical foundations of elasticity. Mineola, NY: Dover, 1994.

[79] Holzapfel, GA. Nonlinear solid mechanics: A continuum approach for engineering. New York, NY: John Wiley \& Sons, 2000.

[80] Gurtin, ME, Fried, E and Anand, L. The mechanics and thermodynamics of continua. Cambridge: Cambridge University Press, 2009.

[81] Toupin, RA. Elastic materials with couple-stresses. Arch Rat Mech Anal 1962; 11(1): 385-414.

[82] Mindlin, R. Second gradient of strain and surface-tension in linear elasticity. Int J Solid Struct 1965; 1(4): $417-438$.

[83] Suiker, ASJ, and Chang CS. Application of higher-order tensor theory for formulating enhanced continuum models. Acta Mech 2000; 142(1-4): 223-234.

[84] Forestm, S, and Aifantism EC. Some links between recent gradient thermo-elasto-plasticity theories and the thermomechanics of generalized continua. Int J Solid Struct 2010; 47(25-26): 3367-3376.

[85] Dell'Isola, F, and Steigmann, D. A two-dimensional gradient-elasticity theory for woven fabrics. J Elast 2014; 118 (1): $113-125$.

[86] Rudraraju, S, Van Der Ven, A, and Garikipati, K. Three-dimensional iso-geometric solutions to general boundary value problems of Toupin's gradient elasticity theory at finite strains. Comput Meth Appl Mech Eng 2014; 278: 705-728.

[87] Carcaterra, A, Dell'Isola, F, Esposito, R, et al. Macroscopic description of microscopically strongly inhomogenous systems: A mathematical basis for the synthesis of higher gradients metamaterials. Arch Rat Mech Anal 2015; 218(3): 1239-1262.

[88] Auffray, N, Dell'Isola, F, Eremeyev, VA et al. Analytical continuum mechanics á la Hamilton-Piola least action principle for second gradient continua and capillary fluids. Math Mech Solid 2015; 20(4): 375-417.

[89] Dell'Isola, F, Giorgio, I, Pawlikowski, M, et al. Large deformations of planar extensible beams and pantographic lattices: Heuristic homogenization, experimental and numerical examples of equilibrium. Proc R Soc A Math Phys Eng Sci 2016; 472(2185): 20150790.

[90] Andreaus, U, Dell'Isola, F, Giorgio, I, et al. Numerical simulations of classical problems in two-dimensional (non) linear second gradient elasticity. Int J Eng Sci 2016; 108: 34-50.

[91] Gurtin, ME. An introduction to continuum mechanics. New York, NY: Academic Press, Inc., 1981.

[92] Podio-Guidugli, P. Primer in elasticity. J Elast 2000; 58(1): 1-104.

[93] Dell'Isola, F, Andreaus, U, and Placidi, L. At the origins and in the vanguard of peridynamics, non-local and higher-gradient continuum mechanics: An underestimated and still topical contribution of Gabrio Piola. Math Mech Solid 2015; 20(8): 887-928.

[94] Ogden, R. Large deformation isotropic elasticity - On the correlation of theory and experiment for incompressible rubberlike solids. Proc R Soc A 1972; 326(1567): 565-584.

[95] Spencer, A. Part III: Theory of invariants. In: Eringen, AC (ed.) Mathematics. New York, NY: Academic Press, 1971, $239-353$.

[96] Spencer, AJM, and Hashin, Z. Continuum theory of the mechanics of fibre-reinforced composites, vol. 53. New York, NY: Springer, 1986.

[97] Javili, A, Steinmann, P, and Mosler, J. Micro-to-macro transition accounting for general imperfect interfaces. Comput Meth Appl Mech Eng 2017; 317: 274-317.

[98] Chatzigeorgiou, G, Meraghni, F, and Javili, A. Generalized interfacial energy and size effects in composites. J Mech Phys Solid 2017; 106: 257-282.

[99] Bowen, RM, and Wang, CC. Introduction to vectors and tensors: Linear and multilinear algebra. New York, NY: Plenum Press, 1976. 
[100] Kreyszig, E. Differential geometry. Mineola, NY: Dover, 1991

[101] Ciarlet, PG. An introduction to differential geometry with applications to elasticity. New York, NY: Springer, 2005.

[102] Steinmann, P. Geometrical foundations of continuum mechanics: An application to first-and second-order elasticity and elastoplasticity. New York, NY: Springer, 2015.

\section{Appendix I Geometry of interfaces}

Basic terminologies and concepts on the differential geometry of interfaces in the sense of two-dimensional manifolds in three-dimensional space are briefly reviewed here; see for example [99-102] for further details. The notation and definitions here correspond to the material configuration keeping in mind that their spatial counterparts are formally identical. A two-dimensional (smooth) interface $\mathcal{I}_{0}$ in the three-dimensional embedding Euclidean space $\mathcal{E}^{3}$ with coordinates $\bar{X}$ can be parameterized by two coordinates $\bar{\eta}^{\alpha}$ with $\alpha=1,2$ as $\overline{\boldsymbol{X}}=\overline{\boldsymbol{X}}\left(\bar{\eta}^{1}, \bar{\eta}^{2}\right)$. The corresponding tangent vectors $\overline{\boldsymbol{G}}_{\alpha} \in T \mathcal{I}_{0}$ to the interface coordinate lines $\bar{\eta}^{\alpha}$, that is, the co-variant (natural) interface basis vectors, are given by $\overline{\boldsymbol{G}}_{\alpha}=\partial_{\bar{\eta}} \overline{\boldsymbol{X}}$ as shown in Figure 9. The associated contra-variant interface basis vectors $\overline{\boldsymbol{G}}^{\alpha}$ are defined by the Kronecker property $\delta_{\beta}^{\alpha}=\overline{\boldsymbol{G}}^{\alpha} \cdot \overline{\boldsymbol{G}}_{\beta}$ and are explicitly related to the co-variant interface basis vectors $\overline{\boldsymbol{G}}_{\alpha}$ by the co- and contra-variant interface metric coefficients $\bar{G}_{\alpha \beta}$ (first fundamental form of the interface) and $\bar{G}^{\alpha \beta}$, respectively, as

$$
\overline{\boldsymbol{G}}_{\alpha}=\bar{G}_{\alpha \beta} \overline{\boldsymbol{G}}^{\beta} \quad \text { with } \quad \bar{G}_{\alpha \beta}=\overline{\boldsymbol{G}}_{\alpha} \cdot \overline{\boldsymbol{G}}_{\beta}=\left[\bar{G}^{\alpha \beta}\right]^{-1} \quad \text { and } \quad \overline{\boldsymbol{G}}^{\alpha}=\bar{G}^{\alpha \beta} \overline{\boldsymbol{G}}_{\beta} \quad \text { with } \quad \bar{G}^{\alpha \beta}=\overline{\boldsymbol{G}}^{\alpha} \cdot \overline{\boldsymbol{G}}^{\beta}=\left[\bar{G}_{\alpha \beta}\right]^{-1} \text {. }
$$

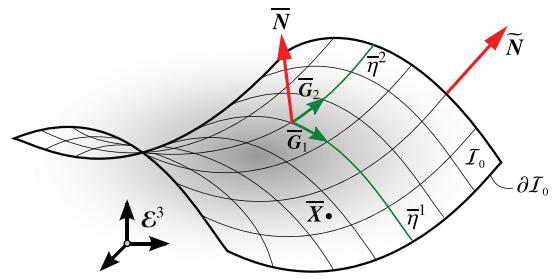

Figure 9. The key differential geometry concepts of the interface as a two-dimensional manifold in three-dimensional embedding Euclidean space $\mathcal{E}^{3}$. Coordinates $\overline{\boldsymbol{X}}$ can be parameterized by two coordinates $\bar{\eta}^{1}$ and $\bar{\eta}^{2}$ as $\overline{\boldsymbol{X}}=\overline{\boldsymbol{X}}\left(\bar{\eta}^{1}, \bar{\eta}^{2}\right)$. The covariance interface tangent vectors are denoted $\overline{\mathbf{G}}_{1}$ and $\overline{\mathbf{G}}_{2}$. The unit normal to the interface is denoted $\overline{\mathbf{N}}$. The outward unit normal to the boundary of the interface and tangential to the interface is denoted $\tilde{\mathbf{N}}$.

The base vectors $\overline{\boldsymbol{G}}^{3}$ and $\overline{\boldsymbol{G}}_{3}$, normal to $T \mathcal{I}_{0}$, are defined by $\overline{\boldsymbol{G}}^{3}:=\overline{\boldsymbol{G}}_{1} \times \overline{\boldsymbol{G}}_{2}$ and $\overline{\boldsymbol{G}}_{3}:=\left[\bar{G}^{33}\right]^{-1} \overline{\boldsymbol{G}}^{3}$ so that $\overline{\boldsymbol{G}}^{3} \cdot \overline{\boldsymbol{G}}_{3}=1$. Thereby, the corresponding contra- and co-variant metric coefficients, respectively, $\left[\bar{G}^{33}\right]$ and $\left[\bar{G}_{33}\right]$, follow as $\left[\bar{G}^{33}\right]=\left|\overline{\boldsymbol{G}}_{1} \times \overline{\boldsymbol{G}}_{2}\right|^{2}=\overline{\operatorname{Det}}\left[\bar{G}_{\alpha \beta}\right]=\left[\overline{\operatorname{Det}}\left[\bar{G}^{\alpha \beta}\right]\right]^{-1}=\left[\bar{G}_{33}\right]^{-1}$. Thus, the interface area element $\mathrm{d} A$ and the interface normal $\overline{\boldsymbol{N}}$ are computed as $\mathrm{d} A=\left|\overline{\boldsymbol{G}}_{1} \times \overline{\boldsymbol{G}}_{2}\right| \mathrm{d} \bar{\eta}^{1} \mathrm{~d} \bar{\eta}^{2}=\left[\bar{G}^{33}\right]^{1 / 2} \mathrm{~d} \bar{\eta}^{1} \mathrm{~d} \bar{\eta}^{2}$ with $\overline{\boldsymbol{N}}=\left[\bar{G}_{33}\right]^{1 / 2} \overline{\boldsymbol{G}}^{3}=\left[\bar{G}^{33}\right]^{1 / 2} \overline{\boldsymbol{G}}_{3}$. Moreover, with $\boldsymbol{I}$ denoting the ordinary mixed-variant unit tensor of the threedimensional embedding Euclidean space, the mixed-variant interface unit tensor $\overline{\boldsymbol{I}}$ is defined as

$$
\overline{\boldsymbol{I}}:=\delta_{\beta}^{\alpha} \overline{\boldsymbol{G}}_{\alpha} \otimes \overline{\boldsymbol{G}}^{\beta}=\overline{\boldsymbol{G}}_{\alpha} \otimes \overline{\boldsymbol{G}}^{\alpha}=\boldsymbol{I}-\overline{\boldsymbol{G}}_{3} \otimes \overline{\boldsymbol{G}}^{3}=\boldsymbol{I}-\overline{\boldsymbol{N}} \otimes \overline{\boldsymbol{N}} .
$$

The interface gradient and interface divergence of a vector field $\{\bullet\}$ are defined by

$$
\overline{\operatorname{Grad}}\{\bullet\}:=\partial_{\bar{\eta}^{\alpha}}\{\bullet\} \otimes \overline{\boldsymbol{G}}^{\alpha}, \quad \overline{\operatorname{Div}}\{\bullet\}:=\partial_{\bar{\eta}^{\alpha}}\{\bullet\} \cdot \overline{\boldsymbol{G}}^{\alpha} .
$$

As a consequence, observe that $\overline{\operatorname{Grad}}\{\bullet\} \cdot \overline{\boldsymbol{N}}=\mathbf{0}$ holds by definition. For fields that are smooth in a neighborhood of the interface, the interface gradient and interface divergence operators are alternatively defined as

$$
\overline{\operatorname{Grad}}\{\bullet\}:=\operatorname{Grad}\{\bullet\} \cdot \overline{\boldsymbol{I}}, \quad \overline{\operatorname{Div}}\{\bullet\}:=\overline{\operatorname{Grad}}\{\bullet\}: \overline{\boldsymbol{I}}=\operatorname{Grad}\{\bullet\}: \overline{\boldsymbol{I}} .
$$

The interface determinant of a second-order tensor field $\{\bullet\}$ is defined by

$$
\overline{\operatorname{Det}}\{\bullet\}:=\frac{\left|\left[\{\bullet\} \cdot \overline{\boldsymbol{G}}_{1}\right] \times\left[\{\bullet\} \cdot \overline{\boldsymbol{G}}_{2}\right]\right|}{\left|\overline{\boldsymbol{G}}_{1} \times \overline{\boldsymbol{G}}_{2}\right|} .
$$


Finally, the derivatives of the co- and contra-variant interface basis vectors read

$$
\partial_{\bar{\eta}^{\beta}} \overline{\boldsymbol{G}}_{\alpha}=\bar{\Gamma}_{\alpha \beta}^{\gamma} \overline{\boldsymbol{G}}_{\gamma}+\bar{K}_{\alpha \beta} \overline{\boldsymbol{N}}, \quad \partial_{\bar{\eta}^{\beta}} \overline{\boldsymbol{G}}^{\alpha}=-\bar{\Gamma}_{\beta \gamma}^{\alpha} \overline{\boldsymbol{G}}^{\gamma}+\bar{K}_{\beta}^{\alpha} \overline{\boldsymbol{N}},
$$

where $\bar{\Gamma}_{\alpha \beta}^{\gamma}=\partial_{\bar{\eta}^{\beta}} \overline{\boldsymbol{G}}_{\alpha} \cdot \overline{\boldsymbol{G}}^{\gamma}$ denote the interface Christoffel symbols and $\bar{K}_{\alpha \beta}$ are the coefficients of the curvature tensor. The curvature tensor $\overline{\boldsymbol{K}}=\bar{K}_{\alpha \beta} \overline{\boldsymbol{G}}^{\alpha} \otimes \overline{\boldsymbol{G}}^{\beta}$ and twice the mean ${ }^{3}$ curvature $\bar{K}=\bar{K}_{\alpha}^{\alpha}$ of the interface $\mathcal{I}_{0}$ are defined as the negative interface gradient and interface divergence of the interface normal $\bar{N}$, respectively:

$$
\overline{\boldsymbol{K}}:=-\overline{\operatorname{Grad}} \overline{\boldsymbol{N}}=-\partial_{\bar{\eta}^{\beta}} \overline{\boldsymbol{N}} \otimes \overline{\boldsymbol{G}}^{\beta}, \quad \bar{K}:=-\overline{\operatorname{Div}} \overline{\boldsymbol{N}}=-\partial_{\bar{\eta}^{\beta}} \overline{\boldsymbol{N}} \cdot \overline{\boldsymbol{G}}^{\beta} .
$$

The co-variant coefficients of the curvature tensor (second fundamental form of the interface) are computed by $\bar{K}_{\alpha \beta}=\overline{\boldsymbol{G}}_{\alpha} \cdot \overline{\boldsymbol{K}} \cdot \overline{\boldsymbol{G}}_{\beta}=-\overline{\boldsymbol{G}}_{\alpha} \cdot \partial_{\bar{\eta} \beta} \overline{\boldsymbol{N}}$.

For an arbitrary vector field $\overline{\boldsymbol{\xi}}$ tangential to the interface, that is, $\overline{\boldsymbol{\xi}}=\overline{\boldsymbol{\xi}} \cdot \overline{\boldsymbol{I}}$, the interface divergence theorem reads

$$
\int_{\partial \mathcal{I}_{0}} \overline{\boldsymbol{\xi}} \cdot \widetilde{\boldsymbol{N}} \mathrm{d} L=\int_{\mathcal{I}_{0}} \overline{\operatorname{Div}} \overline{\boldsymbol{\xi}} \mathrm{d} A \quad \text { with } \quad \overline{\boldsymbol{\xi}}=\left[\overline{\boldsymbol{\xi}}^{\alpha} \overline{\boldsymbol{G}}_{\alpha}, \quad \alpha \in\{1,2\},\right.
$$

in which $\widetilde{N}$ is the unit outward normal to the boundary of the interface but tangential to the interface. The interface divergence theorem (51) is formally identical to the classic divergence theorem in the bulk since it is assumed a priori that the vector field $\bar{\xi}$ is tangential to the interface. Nevertheless, it is possible to establish a general format of the interface divergence theorem for an arbitrary vector field $\bar{\xi}$ not necessarily tangential to the interface. In doing so, the vector $\bar{\xi}$ is first decomposed into its tangential and orthogonal contributions according to

$$
\bar{\xi}=\bar{\xi} \cdot \bar{I}+\bar{\xi} \cdot[\bar{N} \otimes \bar{N}]
$$

and secondly, applying the interface divergence operator gives

$$
\overline{\operatorname{Div}} \bar{\xi}=\overline{\operatorname{Div}}(\bar{\xi} \cdot \overline{\boldsymbol{I}})+\overline{\operatorname{Grad}} \bar{\xi}:[\overline{\boldsymbol{N}} \otimes \overline{\boldsymbol{N}}]+\bar{\xi} \cdot \overline{\operatorname{Grad}} \overline{\boldsymbol{N}} \cdot \overline{\boldsymbol{N}}+\overline{\operatorname{Div}} \overline{\boldsymbol{N}} \bar{\xi} \cdot \overline{\boldsymbol{N}}
$$

in which the second and third terms on the right-hand side vanish due to the property $\overline{\operatorname{Grad}}\{\bullet\} \cdot \overline{\boldsymbol{N}}=\mathbf{0}$ that holds by definition. Furthermore, $\overline{\operatorname{Div}} \bar{N}$ is minus twice the mean curvature and, therefore,

$$
\overline{\operatorname{Div}} \bar{\xi}=\overline{\operatorname{Div}}(\bar{\xi} \cdot \overline{\boldsymbol{I}})-\bar{K} \bar{\xi} \cdot \overline{\boldsymbol{N}}
$$

Next, integrating the identity (54) over the interface furnishes

$$
\int_{\mathcal{I}_{0}} \overline{\operatorname{Div}} \bar{\xi} \mathrm{d} A=\int_{\mathcal{I}_{0}} \overline{\operatorname{Div}}(\bar{\xi} \cdot \overline{\boldsymbol{I}}) \mathrm{d} A-\int_{\mathcal{I}_{0}} \bar{K} \overline{\boldsymbol{\xi}} \cdot \overline{\boldsymbol{N}} \mathrm{d} A
$$

Since $\bar{\xi} \cdot \overline{\boldsymbol{I}}$ is tangential to the interface, one can apply the interface divergence theorem (51) to the first integral on the right-hand side and that gives

$$
\int_{\mathcal{I}_{0}} \overline{\operatorname{Div}} \bar{\xi} \mathrm{d} A=\int_{\partial \mathcal{I}_{0}}[\overline{\boldsymbol{\xi}} \cdot \overline{\boldsymbol{I}}] \cdot \widetilde{\boldsymbol{N}} \mathrm{d} L-\int_{\mathcal{I}_{0}} \bar{K} \overline{\boldsymbol{\xi}} \cdot \overline{\boldsymbol{N}} \mathrm{d} A
$$

Note that, without loss of generality, the relation $[\bar{\xi} \cdot \overline{\boldsymbol{I}}] \cdot \tilde{\boldsymbol{N}}=\bar{\xi} \cdot \widetilde{\boldsymbol{N}}$ holds. Therefore, the interface divergence theorem for an arbitrary vector field $\bar{\xi}$ not necessarily tangential to the interface reads

$$
\int_{\partial \mathcal{I}_{0}} \overline{\boldsymbol{\xi}} \cdot \tilde{\boldsymbol{N}} \mathrm{d} L=\int_{\mathcal{I}_{0}} \overline{\operatorname{Div}} \overline{\boldsymbol{\xi}} \mathrm{d} A+\int_{\mathcal{I}_{0}} \bar{K} \overline{\boldsymbol{\xi}} \cdot \overline{\boldsymbol{N}} \mathrm{d} A \quad \text { with } \quad \overline{\boldsymbol{\xi}}=\left[\overline{\boldsymbol{\xi}}^{a} \overline{\boldsymbol{G}}_{a}, \quad a \in\{1,2,3\} .\right.
$$



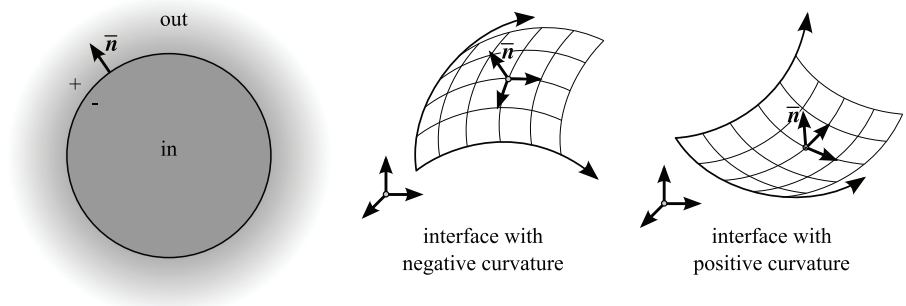

Figure 10. Illustration of an interface between two inviscid fluids satisfying the Young-Laplace equation. The term $\Delta p=p_{\text {in }}-p_{\text {out }}$ is the pressure difference across the interface.

In a near-identical fashion, the interface divergence theorem for an arbitrary second-order tensor field $\bar{\Xi}$ not necessarily tangential to the interface reads

$$
\int_{\partial \mathcal{I}_{0}} \overline{\boldsymbol{\Xi}} \cdot \tilde{\boldsymbol{N}} \mathrm{d} L=\int_{\mathcal{I}_{0}} \overline{\operatorname{Div}} \bar{\Xi} \mathrm{d} A+\int_{\mathcal{I}_{0}} \bar{K} \overline{\boldsymbol{\Xi}} \cdot \overline{\boldsymbol{N}} \mathrm{d} A \quad \text { with } \quad \bar{\Xi}=[\bar{\Xi}]^{a b} \overline{\boldsymbol{G}}_{a} \otimes \overline{\boldsymbol{G}}_{b}, \quad a, b \in\{1,2,3\} .
$$

From the format of equation (58), it is clear that the integral containing the curvature vanishes if the secondorder tensor field $\bar{\Xi}$ is tangential to the interface only with respect to its second index. This particular family of second-order tensors plays an important role in this contribution and they are frequently referred to as superficial tensors [18].

The contents of this section so far correspond to the material configuration. Recall that the quantities and operators in the spatial configuration are distinguished from those in the material configuration using small letters. For instance, the interface identity $\overline{\boldsymbol{I}}=\boldsymbol{I}-\overline{\boldsymbol{N}} \otimes \overline{\boldsymbol{N}}$ in the material configuration is the counterpart of $\overline{\boldsymbol{i}}=\boldsymbol{i}-\overline{\boldsymbol{n}} \otimes \overline{\boldsymbol{n}}$ in the spatial configuration. The full identity tensors $\boldsymbol{I}=\boldsymbol{i}$ are invariant with respect to the configuration, however, this is not the case for the interface identities $\overline{\boldsymbol{I}} \neq \overline{\boldsymbol{i}}$. The interface curvature in the spatial configuration $\bar{k}=-\overline{\operatorname{div}} \overline{\boldsymbol{n}}$ is the counterpart of the curvature in the material configuration $\bar{K}=-\overline{\operatorname{Div}} \overline{\boldsymbol{N}}$ whereby $\overline{\operatorname{div}}\{\bullet\}=\overline{\operatorname{grad}}\{\bullet\}: \overline{\boldsymbol{i}}$.

\section{Appendix 2 Generalized Young-Laplace equation}

The balance equation along the interface $(26)_{3}$ can be understood as a generalized Young-Laplace equation since it reduces to the classical Young-Laplace equation. In order to further clarify the balance equation $(26)_{3}$, the classical example of the Young-Laplace equation is investigated here. The Young-Laplace equation states that the balance equation on the interface between two static fluids reads

$$
\Delta p=\bar{\gamma}\left[\frac{1}{r_{1}}+\frac{1}{r_{2}}\right]
$$

where $\Delta p=p_{\text {in }}-p_{\text {out }}$ is the pressure difference across the fluid interface and $r_{1}$ and $r_{2}$ are the principal radii of curvature. The spatial format of the balance equation along the interface $(26)_{3}$ in the absence of interface force density $\overline{\boldsymbol{b}}_{0}$ reads

$$
\overline{\operatorname{div}} \overline{\boldsymbol{\sigma}}+\llbracket \boldsymbol{\sigma} \rrbracket \cdot \overline{\boldsymbol{n}}=\mathbf{0},
$$

in which $\sigma$ and $\bar{\sigma}$ denote the bulk and interface Cauchy stresses, respectively, associated with the spatial configuration. Considering that $\llbracket \sigma \rrbracket=\sigma_{\text {out }}-\sigma_{\text {in }}$, and employing the relation between the hydrostatic pressure $p$ and Cauchy stress $\sigma$ for inviscid fluids $\sigma=-p \boldsymbol{i}$, results in $\llbracket \sigma \rrbracket=\Delta p \boldsymbol{i}$. Thus, the interface balance (60) reads

$$
\overline{\operatorname{div}} \overline{\boldsymbol{\sigma}}+\Delta p \boldsymbol{i} \cdot \overline{\boldsymbol{n}}=\mathbf{0} \quad \Rightarrow \quad \Delta p \overline{\boldsymbol{n}}=-\overline{\operatorname{div}} \overline{\boldsymbol{\sigma}} .
$$

The interface tension $\bar{\gamma}$ represents a constant energy per unit area in the spatial configuration. Therefore, the internal interface energy density per unit area in the material configuration, corresponding to the interface tension $\bar{\gamma}$, reads $\bar{\psi}_{\text {int }}=\bar{J} \bar{\gamma}$ from which the interface stress $(12)_{1}$ derives as

$$
\overline{\boldsymbol{P}}=\frac{\partial \bar{\psi}_{\text {int }}}{\partial \overline{\boldsymbol{F}}}=\bar{\gamma} \bar{J} \overline{\boldsymbol{F}}^{-\mathrm{t}} \Rightarrow \overline{\boldsymbol{\sigma}}=\bar{J}^{-1} \overline{\boldsymbol{P}} \cdot \overline{\boldsymbol{F}}^{\mathrm{t}}=\bar{\gamma} \overline{\boldsymbol{i}}
$$


where the interface Piola transform $\overline{\boldsymbol{P}}=\overline{\boldsymbol{\sigma}} \cdot \overline{\mathrm{Cof}} \overline{\boldsymbol{F}}$ is utilized. Inserting $\overline{\boldsymbol{\sigma}}=\bar{\gamma} \overline{\boldsymbol{i}}$ into the interface balance (61) yields

$$
\Delta p \overline{\boldsymbol{n}}=-\overline{\operatorname{div}}(\bar{\gamma} \overline{\boldsymbol{i}})=-\bar{\gamma} \overline{\operatorname{div}} \overline{\boldsymbol{i}}=-\bar{\gamma} \bar{k} \overline{\boldsymbol{n}} \Rightarrow \Delta p=-\bar{\gamma} \bar{k},
$$

in which the identity $\overline{\operatorname{div}} \overline{\boldsymbol{i}}=-[\overline{\operatorname{div}} \overline{\boldsymbol{n}}] \overline{\boldsymbol{n}}=\bar{k} \overline{\boldsymbol{n}}$ is used. To obtain the Young-Laplace equation in its classical format, the geometrical definition of the interface curvature

$$
\bar{k}=-\left[\frac{1}{r_{1}}+\frac{1}{r_{2}}\right]
$$

is required, in which the negative sign arises from the convention that the curvature is negative if the interface curves away from its normal, and that the radii of curvatures are always positive; see Figure 10. This example explains how the curvature is embedded within the interface divergence operator. 